\title{
The role of the atomic decoherence-free subalgebra in the study of quantum Markov semigroups
}

Cite as: J. Math. Phys. 60, 072703 (2019); https://doi.org/10.1063/1.5030954

Submitted: 27 March 2018 . Accepted: 22 June 2019 . Published Online: 23 July 2019

Franco Fagnola (D), Emanuela Sasso (D), and Veronica Umanità (D)
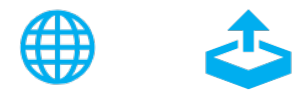

View Online

Export Citation

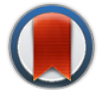

\section{ARTICLES YOU MAY BE INTERESTED IN}

On the moduli spaces of commuting elements in the projective unitary groups

Journal of Mathematical Physics 60, 071703 (2019); https://doi.org/10.1063/1.5097922

Every entangled state provides an advantage in classical communication

Journal of Mathematical Physics 60, 072201 (2019); https://doi.org/10.1063/1.5091856

Where in the world is AIP Publishing?

Find out where we are exhibiting next 


\title{
The role of the atomic decoherence-free subalgebra in the study of quantum Markov semigroups
}

\author{
Cite as: J. Math. Phys. 60, 072703 (2019); doi: 10.1063/1.5030954 \\ Submitted: 27 March 2018 - Accepted: 22 June 2019 • \\ Published Online: 23 July 2019
}

\author{
Franco Fagnola, ${ }^{1, a)}$ (D) Emanuela Sasso, ${ }^{2, b)}$ (D) and Veronica Umanità ${ }^{2}$ \\ AFFILIATIONS \\ ${ }^{1}$ Department of Mathematics, Politecnico di Milano, Piazza Leonardo da Vinci 32, I-20133 Milano, Italy \\ ${ }^{2}$ Dipartimento di Matematica, Università di Genova, Via Dodecaneso 35, 16146 Genova, Italy \\ a) E-mail: franco.fagnola@polimi.it \\ b)E-mail: sasso@dima.unige.it \\ ${ }^{c)}$ E-mail: umanita@dima.unige.it
}

\begin{abstract}
We show that for a Quantum Markov Semigroup (QMS) with a faithful normal invariant state, atomicity of the decoherence-free subalgebra and environmental decoherence are equivalent. Moreover, we prove that the predual of the decoherence-free subalgebra is isometrically isomorphic to the subspace of reversible states. We also describe, in an explicit and constructive way, the relationship between the decoherence-free subalgebra and the fixed point subalgebra.
\end{abstract}

Published under license by AIP Publishing. https://doi.org/10.1063/1.5030954

\section{INTRODUCTION}

Starting from the fundamental papers of Gorini-Kossakowski-Sudarshan ${ }^{21}$ and Lindblad (GKSL), ${ }^{24}$ the structure of uniformly continuous quantum Markov semigroups (QMSs) $\mathcal{T}=\left(\mathcal{T}_{t}\right)_{t \geq 0}$, or, in the physical terminology, quantum dynamical semigroups, and their generators, has been the object of significant attention (see Refs. 3, 6, 14, and 15 and the references therein).

The increasing interest in mathematical modeling of decoherence, coherent quantum computing, and approach to equilibrium in open quantum systems continues to motivate investigation on special features of QMS. Special attention is paid to subalgebras or subspaces where irreversibility and dissipation disappear (see Refs. 2, 3, 8, 15, 16, 23, and 30 and the references therein). States leaving in such subspaces are promising candidates for storing and manipulating quantum information.

The decoherence-free (DF) subalgebra, where completely positive maps $\mathcal{T}_{t}$ of the semigroup act as automorphisms, and the set of fixed points, which is a subalgebra when there exists a faithful invariant state, also allow us to gain insight into the structure of a QMS, its invariant states, and environment induced decoherence. Indeed, its structure as a von Neumann algebra has important consequences on the structure and the action of the whole QMS. Recently, we showed in Ref. 15 that if the decoherence-free subalgebra of a uniformly continuous QMS is atomic, it induces a decomposition of the system into its noiseless and purely dissipative parts, determining the structure of invariant states, as well as decoherence-free subsystems and subspaces. ${ }^{30}$ In particular, we provided a full description of invariant states extending known ones in the finite dimensional case.

In this paper, we push further the analysis of uniformly continuous QMS with atomic decoherence-free subalgebra and a faithful invariant state proving a number of new results we briefly list and outline below.

1. Environment induced decoherence (Refs. 8, 10, and 12) holds if and only if $\mathcal{N}(\mathcal{T})$ is atomic. In this case, the decoherence-free subalgebra is generated by the set of eigenvectors corresponding to modulus one eigenvalues of the completely positive maps $\mathcal{T}_{t}$, namely, in an equivalent way, by the eigenvectors with a purely imaginary eigenvalue of the generator (Theorem 11). 
2. The decoherence-free subalgebra and the set of the so-called reversible states, i.e., the linear space generated by eigenvectors corresponding to modulus 1 eigenvalues of predual maps $\mathcal{T}_{\star t}$, are in the natural duality of a von Neumann algebra with its predual (Theorem 15). Moreover, Theorem 16 explicitly describes the structure of reversible states.

3. We find a spectral characterization of the decomposition of the fixed point algebra (Theorem 19). Moreover, the exact relationship between $\mathcal{F}(\mathcal{T})$ and $\mathcal{N}(\mathcal{T})$ (Theorems 19 and 21) is established in an explicit and constructive way allowing one to find the structure of each one of them from the structure of the other.

Loosely speaking, one can say that for QMSs with a faithful invariant state, the same conclusions can be drawn replacing finite dimensionality of the system Hilbert space by atomicity of the decoherence-free subalgebra.

Counterexamples (Examples 2 and 3) show that, in general, the above conclusions may fail for QMSs without faithful normal invariant states.

The above results clarify the relationships between the atomicity of the decoherence-free subalgebra, the environmental decoherence, the ergodic decomposition of the trace class operators, and the structure of fixed points.

In particular, the first result implies that the decomposition induced by decoherence coincides with the Jacobs-de-Leeuw-Glicksberg (JDG) splitting. Such decomposition was originally introduced for weakly almost periodic semigroups and generalized to QMSs on von Neumann algebras in Refs. 22 and 25. It is among the most useful tools in the study of the asymptotic behavior of operator semigroups on Banach spaces or von Neumann algebras. Indeed, it provides a decomposition of the space into the direct sum of the space generated by eigenvectors of the semigroup associated with modulus 1 eigenvalues and the remaining space, called stable, consisting of all vectors whose orbits have 0 as a weak cluster point. Here, under suitable conditions, we obtain the convergence to 0 for each vector belonging to the stable space.

On the other hand, the ergodic decomposition of trace class operators (which is a particular case of the JDG splitting applied to the predual of $\mathcal{T}$ ) allows one, for instance, to determine reversible subsystems by spectral calculus. The identification of reversible states, in particular, is an important task in the study of irreversible (Markovian) dynamics because they retain their quantum features that are exploited in quantum computation (see Refs. 3 and 30 and the references therein). More precisely, reversible and invariant states of a quantum channel [acting on $M_{n}(\mathbb{C})$ for some $n>1$ ] allow one to classify kinds of information that the process can preserve. When the space is finite-dimensional and there exists a faithful invariant state, the structure of these states can be explicitly found (see, e.g., Lemma 6 and Section $V$ of Ref. 9 and Theorems 6.12 and 6.16 of Ref. 32) through the decomposition of $\mathcal{N}(\mathcal{T})$ and the algebra of fixed points $\mathcal{F}(\mathcal{T})$ in "block diagonal matrices," i.e., in their canonical form given by the structure theorem for matrix algebras (see Theorem 11.2 of Ref. 29). Since a similar decomposition holds for atomic von Neumann algebras, in this paper, we generalize these results to uniformly continuous QMSs acting on $\mathcal{B}(\mathrm{h})$ with $\mathrm{h}$ infinite-dimensional.

The paper is organized as follows. In Sec. II, we collect some notation and known results on the structure of norm-continuous QMS with atomic decoherence-free subalgebra and the structure of their invariant states. In Sec. III, after recalling some known results from Ref. 12 on the relationship between environment induced decoherence (EID) and Jacobs-de Leeuw-Glicksberg decomposition, we prove the main result of this paper: the equivalence between EID and atomicity of the decoherence free subalgebra. The predual algebra of the decoherence-free subalgebra is characterized in Sec. IV as the set of reversible states. Finally, in Sec. V, we study the structure of the set of fixed points of the semigroup and its relationships with the decomposition of $\mathcal{N}(\mathcal{T})$ when this algebra is atomic.

\section{THE STRUCTURE OF THE DECOHERENCE-FREE ALGEBRA}

Let $\mathrm{h}$ be a complex separable Hilbert space, and let $\mathcal{B}(\mathrm{h})$ be the algebra of all bounded operators on $\mathrm{h}$ with unit $\mathbb{1}$. A QMS on $\mathcal{B}(\mathrm{h})$ is a semigroup $\mathcal{T}=\left(\mathcal{T}_{t}\right)_{t \geq 0}$ of completely positive, identity preserving normal maps which is also weakly ${ }^{*}$ continuous. In this paper, we assume $\mathcal{T}$ is uniformly continuous, i.e.,

$$
\lim _{t \rightarrow 0^{+}} \sup _{\|x\| \leq 1}\left\|\mathcal{T}_{t}(x)-x\right\|=0
$$

so that there exists a linear bounded operator $\mathcal{L}$ on $\mathcal{B}(\mathrm{h})$ such that $\mathcal{T}_{t}=e^{t \mathcal{L}}$. The operator $\mathcal{L}$ is the generator of $\mathcal{T}$, and it can be represented in the well-known (see Ref. 27) Gorini-Kossakowski-Sudarshan-Lindblad (GKSL) form as

$$
\mathcal{L}(x)=\mathrm{i}[H, x]-\frac{1}{2} \sum_{\ell \geq 1}\left(L_{\ell}^{*} L_{\ell} x-2 L_{\ell}^{*} x L_{\ell}+x L_{\ell}^{*} L_{\ell}\right)
$$

where $H=H^{*}$ and $\left(L_{\ell}\right)_{\ell \geq 1}$ are bounded operators on $\mathrm{h}$ such that the series $\sum_{\ell \geq 1} L_{\ell}^{*} L_{\ell}$ is strongly convergent and [., $\left.\cdot\right]$ denotes the commutator $[x, y]=x y-y x$. The choice of operators $H$ and $\left(L_{\ell}\right)_{\ell \geq 1}$ is not unique, but this will not create any inconvenience in this paper. More precisely, we have the following characterization (see Proposition 30.14 and Theorem 30.16 of Ref. 27).

Theorem 1. Let $\mathcal{L}$ be the generator of a uniformly continuous $Q M S$ on $\mathcal{B}(\mathrm{h})$. Then, there exist a bounded self-adjoint operator $H$ and a sequence $\left(L_{\ell}\right)_{\ell \geq 1}$ of elements in $\mathcal{B}(h)$ such that 
1. $\sum_{\ell \geq 1} L_{\ell}^{*} L_{\ell}$ is strongly convergent,

2. if $\sum_{\ell \geq 0}\left|c_{\ell}\right|^{2}<\infty$ and $c_{0} \mathbb{1}+\sum_{\ell \geq 1} c_{\ell} \mathrm{L}_{\ell}=0$ for scalars $\left(c_{\ell}\right)_{\ell \geq 0}$, then $\mathrm{c}_{\ell}=0$ for every $\ell \geq 0$,

3. $\mathcal{L}(x)=\mathrm{i}[H, x]-\frac{1}{2} \sum_{\ell \geq 1}\left(L_{\ell}^{*} L_{\ell} x-2 L_{\ell}^{*} x L_{\ell}+x L_{\ell}^{*} L_{\ell}\right)$ for all $x \in \mathcal{B}(\mathrm{h})$.

We recall that for an arbitrary von Neumann algebra $\mathcal{M}$, its predual space $\mathcal{M}_{\star}$ is the space of $w^{*}$-continuous functionals on $\mathcal{M}$ (also called normal). It is a well-known fact that for all $\omega \in \mathcal{M}_{*}$, there exists $\rho \in \mathfrak{I}(\mathrm{h})$, the space of trace-class operators, such that $\omega(x)=\operatorname{tr}(\rho x)$ for all $x \in \mathcal{M}$. In particular, if $\omega$ is a positive and $\|\omega\|=1$, it is called state, and $\rho$ is positive with $\operatorname{tr}(\rho)=1$, i.e., $\rho$ is a density.

If $\mathcal{M}=\mathcal{B}(\mathrm{h})$, every normal state $\omega$ has a unique density $\rho$. Therefore, in this case, we can identify them. Finally, $\rho$ is faithful if $\operatorname{tr}(\rho x)=0$ for a positive $x \in \mathcal{B}(h)$ implies $x=0$ (see Definition 9.4 of Ref. 29).

Given a $w^{*}$-continuous operator $\mathcal{S}: \mathcal{M} \rightarrow \mathcal{M}$, we can define its predual map $\mathcal{S}_{*}: \mathcal{M}_{*} \rightarrow \mathcal{M}_{*}$ as $\mathcal{S}_{*}(\omega)=\omega \circ \mathcal{S}$.

In particular, for $\mathcal{M}=\mathcal{B}(\mathrm{h})$, by considering the predual map of every $\mathcal{T}_{t}$, we obtain the predual semigroup $\mathcal{T}_{*}=\left(\mathcal{T}_{* t}\right)_{t}$ satisfying

$$
\operatorname{tr}\left(\mathcal{T}_{* t}(\rho) x\right)=\operatorname{tr}\left(\rho \mathcal{T}_{t}(x)\right) \quad \forall \rho \in \Im(h), x \in \mathcal{B}(\mathrm{h}) .
$$

The decoherence-free (DF) subalgebra of $\mathcal{T}$ is defined by

$$
\mathcal{N}(\mathcal{T})=\left\{x \in \mathcal{B}(\mathrm{h}): \mathcal{T}_{t}\left(x^{*} x\right)=\mathcal{T}_{t}(x)^{*} \mathcal{T}_{t}(x), \mathcal{T}_{t}\left(x x^{*}\right)=\mathcal{T}_{t}(x) \mathcal{T}_{t}(x)^{*} \forall t \geq 0\right\} .
$$

It is a well known fact that $\mathcal{N}(\mathcal{T})$ is the biggest von Neumann subalgebra of $\mathcal{B}(\mathrm{h})$ on which every $\mathcal{T}_{t}$ acts as a $*$-homomorphism (see, e.g., Theorem 3.1 of Evans ${ }^{18}$ ). Moreover, the following facts hold (see Proposition 2.1 of Ref. 16).

Proposition 2. Let $\mathcal{T}$ be a $\mathrm{QMS}$ on $\mathcal{B}(\mathrm{h})$, and let $\mathcal{N}(\mathcal{T})$ be the set defined by (2). Then,

1. $\mathcal{N}(\mathcal{T})$ is invariant with respect to every $\mathcal{T}_{t}$,

2. $\mathcal{N}(\mathcal{T})=\left\{\delta_{H}^{n}\left(L_{k}\right), \delta_{H}^{n}\left(L_{k}^{*}\right): n \geq 0\right\}^{\prime}$, where $\delta_{\mathrm{H}}(\mathrm{x}):=[\mathrm{H}, \mathrm{x}]$,

3. $\mathcal{T}_{t}(x)=e^{\mathrm{i} t H} x e^{-\mathrm{i} t H}$ for all $x \in \mathcal{N}(\mathcal{T}), \mathrm{t} \geq 0$,

4. if $\mathcal{T}$ possesses a faithful normal invariant state, then $\mathcal{N}(\mathcal{T})$ contains the set of fixed points $\mathcal{F}(\mathcal{T})=\left\{L_{k}, L_{k}^{*}, H: k \geq 1\right\}^{\prime}$.

In addition, since we suppose that $\mathcal{T}$ is uniformly continuous, its action on $\mathcal{N}(\mathcal{T})$ is bijective.

Theorem 3. If $\mathcal{T}$ is a uniformly continuous $Q M S$, then $\mathcal{N}(\mathcal{T})$ is the biggest von Neumann subalgebra on which every map $\mathcal{T}_{t}$ acts as a *-automorphism.

Proof. The restriction of every $\mathcal{T}_{t}$ to $\mathcal{N}(\mathcal{T})$ is clearly injective thanks to item 3 of Proposition 2. Now, given $x \in \mathcal{N}(\mathcal{T})$ and $t>0$, we have to prove that $x=\mathcal{T}_{t}(y)$ for some $y \in \mathcal{N}(\mathcal{T})$.

First of all, note that since the QMS is norm continuous, it can be extended to norm continuous group $\left(\mathcal{T}_{t}\right)_{-\infty<t<+\infty}$ of normal maps on $\mathcal{B}(\mathrm{h})$, and by analyticity in $t, \mathcal{T}_{-t}(z) \in \mathcal{N}(\mathcal{T})$ for all $t>0$ and $z \in \mathcal{N}(\mathcal{T})$, and formula $\mathcal{T}_{-t}(z)=\mathrm{e}^{\mathrm{i} t H} z \mathrm{e}^{\mathrm{i} t H}$ holds.

Remark 4. Notice that Theorem 3 has been proved in Lemma 3.4 of Ref. 22 for a weak ${ }^{*}$ continuous semigroup of positive unital operators satisfying the Schwarz inequality, defined on a von Neumann algebra but possessing a faithful normal invariant state.

As we said in the Introduction, we will see how the atomicity of $\mathcal{N}(\mathcal{T})$ affects fundamental properties of uniformly continuous QMSs.

First of all, as shown in Ref. 15, generalizing the canonical decomposition for matrix algebras, the atomic decoherence-free subalgebra $\mathcal{N}(\mathcal{T})$ (up to an isometric isomorphism) is of the form

$$
\mathcal{N}(\mathcal{T})=\oplus_{i \in I}\left(\mathcal{B}\left(\mathrm{k}_{i}\right) \otimes \mathbb{1}_{\mathrm{m}_{i}}\right)
$$

for some countable sequences of separable Hilbert spaces $\left(\mathrm{k}_{i}\right)_{i \in I}$ and $\left(\mathrm{m}_{i}\right)_{i \in I}$ such that $\mathrm{h}=\oplus_{i \in I}\left(\mathrm{k}_{i} \otimes \mathrm{m}_{i}\right)$ (up to a unitary operator). This structure gives important information on the whole QMS. Let us recall this result.

Theorem 5. Given $\mathcal{N}(\mathcal{T})=\oplus_{i \in I}\left(\mathcal{B}\left(\mathrm{k}_{i}\right) \otimes \mathbb{1}_{\mathrm{m}_{i}}\right)$, the following facts hold:

1. for every $G K S L$ representation of $\mathcal{L}$ by means of operators $H,\left(L_{\ell}\right)_{\ell \geq 1}$, we have

$$
L_{\ell}=\oplus_{i \in I}\left(\mathbb{1}_{\mathrm{k}_{i}} \otimes M_{\ell}^{(i)}\right)
$$


for a collection $\left(M_{\ell}^{(i)}\right)_{\ell \geq 1}$ of operators in $\mathcal{B}\left(\mathrm{m}_{i}\right)$ such that the series $\sum_{\ell \geq 1} M_{\ell}^{(i) *} M_{\ell}^{(i)}$ is strongly convergent for all $i \in I$ and

$$
H=\oplus_{i \in I}\left(K_{i} \otimes \mathbb{1}_{\mathrm{m}_{i}}+\mathbb{1}_{\mathrm{k}_{i}} \otimes M_{0}^{(i)}\right)
$$

for self-adjoint operators $K_{i} \in \mathcal{B}\left(\mathrm{K}_{i}\right)$ and $M_{0}^{(i)} \in \mathcal{B}\left(\mathrm{m}_{i}\right), i \in I$,

2. defining on $\mathcal{B}\left(\mathrm{m}_{i}\right)$, the GKSL generator $\mathcal{L}^{\mathrm{m}_{i}}$ associated with operators $\left.\left\{M_{0}^{(i)}, M_{\ell}^{(i)}\right): \ell^{(i)} \geq 1\right\}$, we have

$$
\mathcal{T}_{t}\left(x_{i} \otimes y_{i}\right)=\mathrm{e}^{\mathrm{i} t K_{i}} x_{i} \mathrm{e}^{-\mathrm{i} t K_{i}} \otimes \mathcal{T}^{\mathrm{m}_{i}}\left(y_{i}\right)
$$

for all $t \geq 0, x_{i} \in \mathcal{B}\left(\mathrm{k}_{i}\right)$, and $y_{i} \in \mathcal{B}\left(\mathrm{m}_{i}\right)$, where $\mathcal{T}^{\mathrm{m}_{i}}$ is the $\mathrm{QMS}$ generated by $\mathcal{L}^{\mathrm{m}_{i}}$,

3. if there exists a faithful normal invariant state, then the QMS $\mathcal{T}^{\mathrm{m}_{i}}$ is irreducible and possesses a unique invariant state $\tau_{\mathrm{m}_{i}}$ which is also faithful, and we have $\mathcal{F}\left(\mathcal{T}^{\mathrm{m}_{i}}\right)=\mathcal{N}\left(\mathcal{T}^{\mathrm{m}_{i}}\right)=\mathbb{C} \mathbb{1}_{\mathrm{m}_{i}}$. Moreover, for all $i \in I, K_{i}$ has a pure point spectrum.

Now, we recall a characterization of an atomic von Neumann algebra in terms of the existence of a normal conditional expectation, i.e., a weakly* ${ }^{*}$-continuous norm one projection (see Theorem 5 of Ref. 31 and Theorem iv.2.2.2 of Ref. 7).

Theorem 6. Let $\mathcal{M}$ be a von Neumann algebra acting on the Hilbert space $h$. Then, $\mathcal{M}$ is atomic if and only if $\mathcal{M}$ is the image of a normal conditional expectation $\mathcal{E}: \mathcal{B}(\mathrm{h}) \rightarrow \mathcal{M}$.

Corollary 7. Let $\mathcal{M}$ be an atomic von Neumann algebra acting on $h$, and let $\mathcal{N} \subseteq \mathcal{M}$ be a von Neumann subalgebra. If there exists a normal conditional expectation $\mathcal{E}: \mathcal{M} \rightarrow \mathcal{N}$ onto $\mathcal{N}$, then $\mathcal{N}$ is atomic.

Proof. By Theorem 6, we know that since $\mathcal{M}$ is atomic, it is the image of a normal conditional expectation $\mathcal{F}: \mathcal{B}(\mathrm{h}) \rightarrow \mathcal{M}$. Therefore, the map $\mathcal{E} \circ \mathcal{F}: \mathcal{B}(h) \rightarrow \mathcal{N}$ is a normal conditional expectation onto $\mathcal{N}$. Indeed, since $\mathcal{N}=\operatorname{Ran} \mathcal{E}$ is contained in $\mathcal{M}=\operatorname{Ran} \mathcal{F}$, for $x \in \mathcal{B}(\mathrm{h})$, we have

$$
(\mathcal{E} \circ \mathcal{F})(\mathcal{E} \circ \mathcal{F}) x=\mathcal{E}^{2}(\mathcal{F}(x))=(\mathcal{E} \circ \mathcal{F})(x),
$$

i.e., $\mathcal{E} \circ \mathcal{F}$ is a projection. Therefore, $\|\mathcal{E} \circ \mathcal{F}\| \geq 1$. On the other hand, since $\mathcal{E}$ and $\mathcal{F}$ are norm one operators, we clearly obtain $\|\mathcal{E} \circ \mathcal{F}\|=1$. The normality of $\mathcal{E} \circ \mathcal{F}$ is evident, and so we can conclude that the algebra $\mathcal{N}$ is atomic by Tomiyama theorem.

Remark 8. Theorem 6 is a simplified version of Tomiyama theorem (Theorem 5 of Ref. 31), given in terms of atomicity of the subalgebra $\mathcal{M}$. Moreover, Corollary 7 generalizes to atomic algebras one implication of the same theorem. In particular, we give easier proofs of these results.

In the following, we assume the existence of a faithful normal invariant state; note that, in general, this condition is not necessary for the decoherence-free subalgebra to be atomic. This is always the case for any QMS acting on a finite dimensional algebra. However, we show the following example that will be useful later. by

Example 1. Let $\mathrm{h}=\mathbb{C}^{3}$ with the canonical orthonormal basis $\left(e_{i}\right)_{i=1,2,3}$ and $\mathcal{B}(\mathrm{h})=M_{3}(\mathbb{C})$. We consider the operator $\mathcal{L}$ on $M_{3}(\mathbb{C})$ given

$$
\mathcal{L}(x)=\mathrm{i} \omega\left[\left|e_{1}\right\rangle\left\langle e_{1}\right|, x\right]-\frac{1}{2}\left(\left|e_{3}\right\rangle\left\langle e_{3}|x-2| e_{3}\right\rangle\left\langle e_{2}|x| e_{2}\right\rangle\left\langle e_{3}|+x| e_{3}\right\rangle\left\langle e_{3}\right|\right)
$$

for all $x \in M_{3}(\mathbb{C})$, with $\omega \in \mathbb{R}, \omega \neq 0$. Clearly, $\mathcal{L}$ is written in the GKSL form with $H=\omega\left|\mathrm{e}_{1}\right\rangle\left\langle\mathrm{e}_{1}\right|$ and $L=\left|\mathrm{e}_{2}\right\rangle\left\langle\mathrm{e}_{3}\right|$, and so it generates a uniformly continuous QMS $\mathcal{T}=\left(\mathcal{T}_{t}\right)_{t \geq 0}$ on $M_{3}(\mathbb{C})$.

An easy computation shows that any invariant functional has the form

$$
a\left|e_{1}\right\rangle\left\langle e_{1}|+b| e_{2}\right\rangle\left\langle e_{2}\right|
$$

for some $a, b \in \mathbb{C}$, and so the semigroup has no faithful invariant states.

Since $[H, L]=0$, by item 2 of Proposition 2, we have $\mathcal{N}(\mathcal{T})=\left\{L, L^{*}\right\}^{\prime}$ so that an element $x \in \mathcal{B}(\mathrm{h})$ belongs to $\mathcal{N}(\mathcal{T})$ if and only if

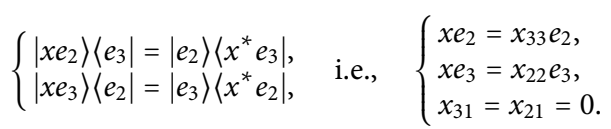


Therefore, we get

$$
\mathcal{N}(\mathcal{T})=\left\{x_{11}\left|e_{1}\right\rangle\left\langle e_{1}\left|+x_{22}\left(\left|e_{2}\right\rangle\left\langle e_{2}|+| e_{3}\right\rangle\left\langle e_{3}\right|\right)\right| x_{11}, x_{22} \in \mathbb{C}\right\},\right.
$$

i.e., $\mathcal{N}(\mathcal{T})$ is isometrically isomorphic to the atomic algebra $\mathbb{C} \oplus \mathbb{C} p$, where $p$ denotes the identity matrix in $M_{2}(\mathbb{C})$.

\section{Atomicity of $\mathcal{N}(\mathcal{T})$ and decoherence}

In this section, we explore the relationships between the atomicity of $\mathcal{N}(\mathcal{T})$ and the property of environmental decoherence, assuming the existence of a faithful normal invariant state $\rho$. Following Ref. 12, we say that there is environment induced decoherence (EID) on the open system described by $\mathcal{T}$ if there exists a $\mathcal{T}_{t}$-invariant and ${ }^{*}$-invariant weakly ${ }^{*}$ closed subspace $\mathcal{M}_{2}$ of $\mathcal{B}(\mathrm{h})$ such that

(EID1) $\mathcal{B}(\mathrm{h})=\mathcal{N}(\mathcal{T}) \oplus \mathcal{M}_{2}$ with $\mathcal{M}_{2} \neq\{0\}$

(EID2) $\quad w^{*}-\lim _{t \rightarrow \infty} \mathcal{T}_{t}(x)=0$ for all $x \in \mathcal{M}_{2}$.

Unfortunately, if EID holds and $\mathrm{h}$ is infinite-dimensional, it is not clear if the space $\mathcal{M}_{2}$ is uniquely determined. However, $\mathcal{M}_{2}$ is always contained in the $\mathcal{T}$-invariant and $*$-invariant closed subspace

$$
\mathcal{M}_{0}=\left\{x \in \mathcal{B}(\mathrm{h}): w^{*}-\lim _{t \rightarrow \infty} \mathcal{T}_{t}(x)=0\right\}
$$

In Ref. 15, we showed that if $\mathcal{N}(\mathcal{T})$ is atomic, then EID holds (see Theorem 5.1) and, in particular, $\mathcal{N}(\mathcal{T})$ is the image of a normal conditional expectation $\mathcal{E}: \mathcal{B}(\mathrm{h}) \rightarrow \mathcal{N}(\mathcal{T})$ compatible with the faithful state $\rho$ (i.e., $\rho \circ \mathcal{E}=\rho$ ) and such that

$$
\operatorname{Ker} \mathcal{E}=\mathcal{M}_{2}=\{x \in \mathcal{B}(\mathrm{h}): \operatorname{tr}(\rho x y)=0 \forall y \in \mathcal{N}(\mathcal{T})\}
$$

(see Theorem 19 of Ref. 12). More precisely, $\mathcal{E}$ is given by

$$
\mathcal{E}(x)=\sum_{i}\left(\sum_{j} \mathrm{~m}_{i}\left\langle f_{j}\left|\left(\mathbb{1}_{\mathrm{k}_{i}} \otimes \tau_{\mathrm{m}_{i}}\right) x_{i i}\right| f_{j}\right\rangle_{\mathrm{m}_{i}} \otimes \mathbb{1}_{\mathrm{m}_{i}}\right),
$$

where $x_{i i}=p_{i} x p_{i}$ for all $x \in \mathcal{B}(\mathrm{h}), \tau_{\mathrm{m}_{i}}$ is the unique faithful invariant state for $\mathcal{T}^{\mathrm{m}_{i}},\left(f_{j}\right)_{j}$ is an orthonormal basis of $\mathrm{m}_{i}$ of eigenvectors of $\tau_{\mathrm{m}_{i}}$, and

$$
\left|f_{j}\right\rangle_{\mathrm{m}_{i}}: \mathrm{k}_{i} \rightarrow \mathrm{k}_{i} \otimes \mathrm{m}_{i}, \quad\left|f_{j}\right\rangle_{\mathrm{m}_{i}} e=e \otimes f_{j}
$$

with adjoint operators

$$
\mathrm{m}_{i}\left\langle f_{j}\right|: \mathrm{k}_{i} \otimes \mathrm{m}_{i} \rightarrow \mathrm{k}_{i}, \quad \mathrm{~m}_{i}\left\langle f_{j}\right| u \otimes v=\left\langle f_{j}, v\right\rangle u .
$$

In the following, we will show that if $\mathcal{N}(\mathcal{T})$ is atomic, the decomposition induced by decoherence is unique, i.e., the only way to realize it is taking $\mathcal{M}_{2}$ given by (4) (see Theorem 11 and Remark 12.2).

Moreover, in this case, we will study the relationships of such a decomposition with another celebrated asymptotic splitting of $\mathcal{B}(\mathrm{h})$, called the Jacobs-de Leeuw-Glicksberg splitting: this comparison is very natural since the decomposition $\mathcal{B}(\mathrm{h})=\mathcal{N}(\mathcal{T}) \oplus \mathcal{M}_{2}$ is clearly related to the asymptotic properties of the semigroup.

We recall that since there exists $\rho$ faithful invariant, the Jacobs-de Leeuw-Glicksberg splitting holds (see, e.g., Corollary 3.3 and Proposition 3.3 of Ref. 22) and is given by $\mathcal{B}(\mathrm{h})=\mathfrak{M}_{r} \oplus \mathfrak{M}_{s}$ with

$$
\begin{aligned}
& \mathfrak{M}_{r}:=\overline{\operatorname{span}} w^{*}\left\{x \in \mathcal{B}(\mathrm{h}): \mathcal{T}_{t}(x)=e^{\mathrm{it} \lambda} x \text { for some } \lambda \in \mathbb{R}, \forall t \geq 0\right\}, \\
& \mathfrak{M}_{s}:=\left\{x \in \mathcal{B}(\mathrm{h}): 0 \in \overline{\left\{\mathcal{T}_{t}(x)\right\}_{t \geq 0}} w^{*} .\right.
\end{aligned}
$$

Moreover, in this case, $\mathfrak{M}_{r}$ is a von Neumann algebra and there exists a normal conditional expectation onto $\mathfrak{M}_{r}$ compatible with $\rho$.

The relationship between the decomposition induced by decoherence and the Jacobs-de Leeuw-Glicksberg splitting is given by the following result (see Proposition 31 of Ref. 12).

Proposition 9. If there exists a faithful normal invariant state $\rho$, then the following conditions are equivalent:

1. EID holds with $\mathcal{M}_{2}=\mathcal{M}_{0}$, and the induced decomposition coincides with the Jacobs-de Leeuw-Glicksberg splitting,

2. $\mathcal{N}(\mathcal{T}) \cap \mathfrak{M}_{s}=\{0\}$,

3. $\mathcal{N}(\mathcal{T})=\mathfrak{M}_{r}$. 
Moreover, if one of the previous conditions holds, then $\mathcal{N}(\mathcal{T})$ is the image of a normal conditional expectation $\mathcal{E}$ compatible with $\rho$ and such that $\operatorname{Ker} \mathcal{E}=\mathcal{M}_{0}=\mathfrak{M}_{s}$.

Proof. The implication $1 \Rightarrow 2$ is clear since $\mathfrak{M}_{r} \cap \mathfrak{M}_{s}=\{0\}$. Conversely, assume $\mathcal{N}(\mathcal{T}) \cap \mathfrak{M}_{s}=\{0\}$ and let $x \in \mathcal{N}(\mathcal{T})$. Since JDG holds, we have $x=x_{1}+x_{2}$ with $x_{1} \in \mathfrak{M}_{r} \subseteq \mathcal{N}(\mathcal{T})$ and $x_{2} \in \mathfrak{M}_{s}$. It follows that also $x_{2}$ belongs to $\mathcal{N}(\mathcal{T})$, and then $x_{2}=0$. Therefore, $x=x_{1} \in \mathfrak{M}_{r}$. This proves the equivalence of statements 1 and 2 .

$1 \Rightarrow 3$ : Assume $\mathfrak{M}_{r}=\mathcal{N}(\mathcal{T})$, and let $x \in \mathfrak{M}_{s}$. If there exists a weak ${ }^{*}$ cluster point $x_{0} \neq 0$ for $\left\{\mathcal{T}_{t}(x)\right\}_{t \geq 0}$, then $x_{0}$ belongs to $\mathcal{N}(\mathcal{T})=\mathfrak{M}_{r}$. Since $\mathfrak{M}_{r} \cap \mathfrak{M}_{s}=\{0\}$, we obtain $x_{0}=0$. This proves that there exists $w^{*}-\lim _{t} \mathcal{T}_{t}(x)=0$, i.e., $x$ belongs to $\mathcal{M}_{0}$ giving the equality $\mathfrak{M}_{s}=\mathcal{M}_{0}$. Therefore, EID holds with $\mathcal{M}_{2}=\mathfrak{M}_{s}=\mathcal{M}_{0}$, i.e., the decomposition induced by decoherence coincides with the JDG splitting. The opposite implication is trivial.

If one of the conditions (1-3) holds, then $\mathcal{N}(\mathcal{T})$ is the image of a normal conditional expectation $\mathcal{E}$ compatible with $\omega$.

Clearly, if $\mathfrak{M}_{r}$ is not an algebra, it does not make sense to ask oneself if it coincides with $\mathcal{N}(\mathcal{T})$. In particular, this could happen when $\mathcal{T}$ has no faithful invariant states, as the following example shows.

Example 2. Let us consider the uniformly continuous QMS $\mathcal{T}$ on $M_{3}(\mathbb{C})$ defined in Example 1 . We have already seen that $\mathcal{T}$ does not possess faithful invariant states and

$$
\mathcal{N}(\mathcal{T})=\left\{x_{11}\left|e_{1}\right\rangle\left\langle e_{1}\left|+x_{22}\left(\left|e_{2}\right\rangle\left\langle e_{2}|+| e_{3}\right\rangle\left\langle e_{3}\right|\right)\right| x_{11}, x_{22} \in \mathbb{C}\right\} .\right.
$$

Now, we find the space $\mathfrak{M}_{r}$, generated by eigenvectors of $\mathcal{L}$ corresponding to purely imaginary eigenvalues. Easy computations show that we have $\mathcal{L}(x)=i \lambda x$ for some $\lambda \in \mathbb{R}$ if and only if

$$
\begin{aligned}
\mathrm{i} \lambda \sum_{i, j=1}^{3} x_{i j}\left|e_{i}\right\rangle\left\langle e_{j}\right| & =\mathrm{i} \omega \sum_{j=1}^{3}\left(x_{1 j}\left|e_{1}\right\rangle\left\langle e_{j}\left|-x_{j 1}\right| e_{j}\right\rangle\left\langle e_{1}\right|\right) \\
& -\frac{1}{2}\left(\sum_{j=1}^{3} x_{3 j}\left|e_{3}\right\rangle\left\langle e_{j}\left|-2 x_{22}\right| e_{3}\right\rangle\left\langle e_{3}\left|+\sum_{i=1}^{3} x_{i 3}\right| e_{i}\right\rangle\left\langle e_{3}\right|\right),
\end{aligned}
$$

i.e., in the case when $\lambda=0, \mathrm{x}_{\mathrm{ij}}=0$ for $\mathrm{i} \neq \mathrm{j}$ and $\mathrm{x}_{22}=\mathrm{x}_{33}$, and, in the case $\lambda \neq 0$, if and only if the following identities hold:

$$
\begin{array}{ccc}
x_{11}=0, & x_{22}=0, & x_{12}(\omega-\lambda)=0 \\
x_{13}\left(-\frac{1}{2}+\mathrm{i}(\omega-\lambda)\right)=0, & x_{21}(\omega+\lambda)=0, & x_{23}\left(\frac{1}{2}+\mathrm{i} \lambda\right)=0, \\
x_{31}\left(\frac{1}{2}+\mathrm{i}(\omega+\lambda)\right)=0, & x_{32}\left(\frac{1}{2}+\mathrm{i} \lambda\right)=0, & x_{33}(1+\mathrm{i} \lambda)=x_{22} .
\end{array}
$$

Since $\omega$ and $\lambda$ belong to $\mathbb{R}$, this is equivalent to either $x=x_{12}\left|e_{1}\right\rangle\left\langle e_{2}\right|$ and $\lambda=\omega$ or $x=x_{21}\left|e_{2}\right\rangle\left\langle e_{1}\right|$ and $\lambda=-\omega$. Therefore, we can conclude that

$$
\mathfrak{M}_{r}=\left\{\left(\begin{array}{ccc}
x_{11} & x_{12} & 0 \\
x_{21} & x_{22} & 0 \\
0 & 0 & x_{22}
\end{array}\right): x_{11}, x_{22}, x_{12}, x_{21} \in \mathbb{C}\right\} .
$$

In particular, $\mathfrak{M}_{r}$ is strictly bigger than $\mathcal{N}(\mathcal{T})$ and it is not an algebra: indeed, for example,

$$
x=\left(\begin{array}{lll}
1 & 1 & 0 \\
1 & 1 & 0 \\
0 & 0 & 1
\end{array}\right) \in \mathfrak{M}_{r}
$$

but $x^{2} \notin \mathfrak{M}_{r}$.

Remark 10. 1. Note that if $\mathfrak{M}_{r}$ is contained in $\mathcal{N}(\mathcal{T})$, then it is a $*$-algebra.

Indeed, if $\mathfrak{M}_{r} \subseteq \mathcal{N}(\mathcal{T})$, taken $x, y \in \mathfrak{M}_{r}$ such that $\mathcal{T}_{t}(x)=e^{\mathrm{i} \lambda t} x$ and $\mathcal{T}_{t}(y)=e^{\mathrm{i} \mu t} y$ for some $\lambda, \mu \in \mathbb{R}$ and any $t$, by property 3 in Proposition 2 we have

As a consequence, $x^{*} y$ belongs to $\mathfrak{M}_{r}$.

$$
\mathcal{T}_{t}\left(x^{*} y\right)=\mathcal{T}_{t}(x)^{*} \mathcal{T}_{t}(y)=e^{\mathrm{i} t(\mu-\lambda)} x^{*} y \quad \forall t \geq 0
$$

2. If $\mathrm{h}$ is finite-dimensional, then also the opposite implication is true.

Indeed, if $\mathfrak{M}_{r}$ is a $*$-algebra, given $x \in \mathcal{B}(\mathrm{h})$ such that $\mathcal{T}_{t}(x)=e^{i t \lambda} x, \lambda \in \mathbb{R}$, we have $\mathcal{T}_{t}\left(x^{*}\right) \mathcal{T}_{t}(x)=x^{*} x$. Then, by the Schwarz inequality, $\mathcal{T}_{t}\left(x^{*} x\right) \geq x^{*} x$ for all $t \geq 0$. Set $\mathcal{T}_{t}^{r}:=\mathcal{T}_{t \mid \mathfrak{m}_{r}}$. Since in this case $\mathcal{T}$ is a strongly continuous semigroup, by definition of $\mathfrak{M}_{r}$ and by 
Corollary 2.9, Chap. V, of Ref. 17, the strong operator closure of $\left\{\mathcal{T}_{t}^{r}: t \geq 0\right\}$ is a compact topological group of operators in $\mathcal{B}\left(\mathfrak{M}_{r}\right)$. Hence, $\left(\mathcal{T}_{t}^{r}\right)^{-1}$ is the limit of some net $\left(\mathcal{T}_{t_{\alpha}}^{r}\right)_{\alpha}$ and so $\left(\mathcal{T}_{t}^{r}\right)^{-1}$ is a positive operator. Since $x^{*} x \in \mathfrak{M}_{r}$, for all $\alpha$, we have $\mathcal{T}_{t_{\alpha}}^{r}\left(x^{*} x\right) \geq x^{*} x$, and so $\left(\mathcal{T}_{t}^{r}\right)^{-1}\left(x^{*} x\right) \geq x^{*} x$. On the other hand,

$$
\left(\mathcal{T}_{t}^{r}\right)^{-1}\left(\mathcal{T}_{t}^{r}\left(x^{*} x\right)\right)=x^{*} x \geq\left(\mathcal{T}_{t}^{r}\right)^{-1}\left(x^{*} x\right) .
$$

Therefore, $\left(\mathcal{T}_{t}^{r}\right)^{-1}\left(x^{*} x\right)=x^{*} x$ and this implies $\mathcal{T}_{t}\left(x^{*} x\right)=x^{*} x=\mathcal{T}_{t}(x)^{*} \mathcal{T}_{t}(x)$. Similarly, we can prove the equality $\mathcal{T}_{t}\left(x x^{*}\right)=x x^{*}$ $=\mathcal{T}_{t}(x) \mathcal{T}_{t}(x)^{*}$, and so $x$ belongs to $\mathcal{N}(\mathcal{T})$.

It is not yet clear what happens when $\mathrm{h}$ is infinite dimensional.

Now, we are able to prove one of the central results of this paper.

Theorem 11. Assume that there exists a faithful normal invariant state $\rho$. Then, $\mathcal{N}(\mathcal{T})$ is atomic if and only if EID holds with $\mathcal{N}(\mathcal{T})=\mathfrak{M}_{r}$ and $\mathcal{M}_{2}=\mathcal{M}_{0}$.

Proof. If $\mathcal{N}(\mathcal{T})$ is atomic, then EID holds by Theorem 5.1 of Ref. 15 . It remains to prove that $\mathcal{N}(\mathcal{T})=\mathfrak{M}_{r}$ and $\mathcal{M}_{2}=\mathcal{M}_{0}$. The atomicity implies $\mathcal{N}(\mathcal{T})=\oplus_{i \in I}\left(\mathcal{B}\left(\mathrm{k}_{i}\right) \otimes \mathbb{1}_{\mathrm{m}_{i}}\right)$ up to a unitary isomorphism. Let $x=\sum_{i \in I}\left(x_{i} \otimes \mathbb{1}_{\mathrm{m}_{i}}\right)$ be in $\mathcal{N}(\mathcal{T}) \cap \mathfrak{M}_{s}$, with $x_{i} \in \mathcal{B}\left(\mathrm{k}_{i}\right)$ for every $i \in I$, and assume $w^{*}-\lim _{\alpha} \mathcal{T}_{t_{\alpha}}(x)=0$. Given $u_{i}, v_{i} \in \mathrm{K}_{i}$ and $\tau_{i}$ is an arbitrary state on $\mathrm{m}_{i}$, by Theorem 5 ,

$$
\operatorname{tr}\left(\left(\left|u_{i}\right\rangle\left\langle v_{i}\right| \otimes \tau_{i}\right) \mathcal{T}_{t_{\alpha}}(x)\right)=\left\langle v_{i}, e^{\mathrm{i} t_{\alpha} K_{i}} x_{i} e^{-\mathrm{i} t_{\alpha} K_{i}} u_{i}\right\rangle
$$

Choosing $u_{i}$ and $v_{i}$ such that $K_{i} u_{i}=\lambda_{i} u_{i}$ and $K_{i} v_{i}=\mu_{i} v_{i}, \lambda_{i}, \mu_{i} \in \mathbb{R}$, Eq. (8) becomes

$$
\operatorname{tr}\left(\left(\left|u_{i}\right\rangle\left\langle v_{i}\right| \otimes \tau_{i}\right) \mathcal{T}_{t_{\alpha}}(x)\right)=e^{\mathrm{i} t_{\alpha}\left(\mu_{i}-\lambda_{i}\right)}\left\langle v_{i}, x_{i} u_{i}\right\rangle
$$

so that $\left\langle v_{i}, x_{i} u_{i}\right\rangle=0$, i.e., $x_{i}=0$ because the eigenvectors of $K_{i}$ form an orthonormal basis of $\mathrm{k}_{i}$ (see item 3 of Theorem 5 ). This proves the equality $\mathcal{N}(\mathcal{T}) \cap \mathfrak{M}_{s}=\{0\}$.

So we can conclude thanks to item 3 of Proposition 9.

Conversely, if EID holds with $\mathcal{N}(\mathcal{T})=\mathfrak{M}_{r}$ and $\mathcal{M}_{2}=\mathcal{M}_{0}$, by Proposition 9 , there exists a normal conditional expectation $\mathcal{E}: \mathcal{B}(\mathrm{h}) \rightarrow \mathcal{N}(\mathcal{T})$ onto $\mathcal{N}(\mathcal{T})$, compatible to $\rho$. Therefore, $\mathcal{N}(\mathcal{T})$ is atomic thanks to Theorem 6

Remarks 12. As a consequence of Theorem 11 and Proposition 9, the following facts hold:

1. $\mathcal{N}(\mathcal{T})$ is atomic if and only if $\mathcal{N}(\mathcal{T}) \cap \mathfrak{M}_{s}=\{0\}$, if and only if $\mathcal{N}(\mathcal{T})=\mathfrak{M}_{r}$, i.e. $\mathcal{N}(\mathcal{T})$ is generated by eigenvectors of $\mathcal{L}$ corresponding to purely imaginary eigenvalues.

Moreover, in this case, we also have $\mathcal{N}(\mathcal{T}) \cap \mathcal{M}_{0}=\{0\}$ being $\mathcal{M}_{0} \subseteq \mathfrak{M}_{s}$ : this means that assuming $\mathcal{N}(\mathcal{T})$ is atomic and the existence of a faithful invariant state, the situation is similar to the finite-dimensional case, i.e., $\mathcal{N}(\mathcal{T})$ does not contain operators going to 0 under the action of the semigroup.

2. If $\mathcal{N}(\mathcal{T})$ is atomic and $\mathcal{F}(\mathcal{T})=\mathbb{C} 1$, the semigroup satisfies the following properties given by noncommutative Perron-Frobenius theorem (see, e.g., Propositions 6.1 and 6.2 of Ref. 5 and Theorem 2.5 of Ref. 4):

- the peripheral point spectrum $\sigma_{p}\left(\mathcal{T}_{t}\right) \cap \mathbb{T}$ of each $\mathcal{T}_{t}$ is a subgroup of the circle group $\mathbb{T}$,

- given $t \geq 0$, each peripheral eigenvalue $\alpha$ of $\mathcal{T}_{t}$ is simple and we have $\sigma_{p}\left(\mathcal{T}_{t}\right) \cap \mathbb{T}=\alpha\left(\sigma_{p}\left(\mathcal{T}_{t}\right) \cap \mathbb{T}\right)$,

- the restriction of $\rho$ to $\mathcal{N}(\mathcal{T})$ is a trace.

As a consequence, the peripheral point spectrum of each $\mathcal{T}_{t}$ is the cyclic group of all $h$-roots of unit for some $h \in \mathbb{N}$.

3. If $\mathcal{N}(\mathcal{T})$ is atomic, the decomposition induced by decoherence is uniquely determined. This fact follows from Proposition 5 of Ref. 12 since we have $\mathcal{N}(\mathcal{T}) \cap \mathfrak{M}_{0}=\{0\}$.

4. Note that Theorem 11 does not exclude the possibility to have a QMS $\mathcal{T}$ displaying decoherence with $\mathcal{N}(\mathcal{T})$, a nonatomic type I algebra. Clearly, in this case, we will get $\mathcal{N}(\mathcal{T}) \varsubsetneqq \mathfrak{M}_{r}$ or $\mathcal{M}_{2} \mp \mathcal{M}_{0}$.

Remark 13. In Ref. 26, the authors proved that EID holds when the semigroup commutes with the modular group associated with a faithful normal invariant state. However, our result in Theorem 11 is stronger on $\mathcal{B}(\mathrm{h})$ since we find the equivalence between the EID and the atomicity of $\mathcal{N}(\mathcal{T})$, which is a weaker assumption of the commutation with the modular group. In fact, it can be shown 
(see Sec. 3 of Ref. 28) that commutation with the modular group implies atomicity of $\mathcal{N}(\mathcal{T})$. Moreover, it is not difficult to find an example of a QMS on $\mathcal{B}(\mathrm{h})$ with $\mathrm{h}$ finite dimensional which does not commute with the modular group. Its decoherence-free subalgebra, as any finite dimensional von Neumann algebra, will be atomic.

\section{STRUCTURE OF REVERSIBLE STATES}

In this section, assuming $\mathcal{N}(\mathcal{T})$ is atomic and the existence of a faithful invariant state $\rho$, we study the structure of reversible states, i.e., states belonging to the vector space

$$
\begin{aligned}
\mathcal{R}\left(\mathcal{T}_{*}\right): & =\overline{\operatorname{span}}\left\{\sigma \in \mathfrak{I}(\mathrm{h}): \mathcal{T}_{* t}(\sigma)=e^{\mathrm{i} t \lambda} \sigma \text { for some } \lambda \in \mathbb{R}, \forall t \geq 0\right\}, \\
& =\overline{\operatorname{span}}\left\{\sigma \in \mathfrak{I}(\mathrm{h}): \mathcal{L}_{*}(\sigma)=\mathrm{i} \lambda \sigma \text { for some } \lambda \in \mathbb{R}\right\} .
\end{aligned}
$$

In particular, we will prove that $\mathcal{R}\left(\mathcal{T}_{*}\right)$ is the predual of the decoherence-free algebra $\mathcal{N}(\mathcal{T})$.

To this end, we recall the following result which is a version of the Jacobs-de Leeuw-Glicksberg theorem for strongly continuous semigroups (see Propositions 3.1 and 3.2 of Ref. 25 and Theorem 2.8 of Ref. 17).

Theorem 14. If there exists a density $\rho \in \mathfrak{I}(\mathrm{h})$ satisfying

$$
\operatorname{tr}\left(\rho\left(\mathcal{T}_{t}(x)^{*} \mathcal{T}_{t}(x)\right)\right) \leq \operatorname{tr}\left(x^{*} x\right) \quad \forall x \in \mathcal{B}(\mathrm{h}), t \geq 0,
$$

then we can decompose $\mathfrak{I}(\mathrm{h})$ as

$$
\mathfrak{I}(\mathrm{h})=\mathcal{R}\left(\mathcal{T}_{*}\right) \oplus\left\{\sigma \in \mathfrak{I}(\mathrm{h}): 0 \in{\overline{\left\{\mathcal{T}_{\star t}(\sigma)\right.}}_{t \geq 0}^{w}\right\}
$$

Since each faithful invariant state clearly fulfills (11), we obtain the splitting given by Eq. (12).

On the other hand, denoting by $A^{\perp}$, the vector space $\{\sigma \in \mathfrak{I}(\mathrm{h}): \operatorname{tr}(\sigma x)=0 \forall x \in A\}$ for all subset $A$ of $\mathcal{B}(\mathrm{h})$, the atomicity of $\mathcal{N}(\mathcal{T})$ ensures the following facts:

(F1). $\quad \mathcal{B}(\mathrm{h})=\mathcal{N}(\mathcal{T}) \oplus \mathcal{M}_{0}$ with $\mathcal{N}(\mathcal{T})=\mathfrak{M}_{r}=\operatorname{Ran} \mathcal{E}$ and $\mathcal{M}_{0}=\mathfrak{M}_{s}=$ KerE, where $\mathcal{E}: \mathcal{B}(\mathrm{h}) \rightarrow \mathcal{N}(\mathcal{T})$ is a normal conditional expectation compatible with the faithful state $\rho$ (see Theorem 11 and Proposition 9);

(F2). $\quad \Im(h)=\mathcal{M}_{0}^{\perp} \oplus \mathcal{N}(\mathcal{T})^{\perp}$ with

$$
\mathcal{M}_{0}^{\perp}=\operatorname{Ran} \mathcal{E}_{*} \simeq \mathcal{N}(\mathcal{T})_{*}, \quad \mathcal{N}(\mathcal{T})^{\perp}=\operatorname{Ker} \mathcal{E}_{*} \simeq \mathcal{M}_{2 *}
$$

Moreover, each $\mathcal{T}_{* t}$ acts as a surjective isometry on $\mathcal{M}_{0}^{\perp}$, and $w-\lim _{t} \mathcal{T}_{\star t}(\sigma)=0$ for all $\sigma \in \mathcal{N}(\mathcal{T})^{\perp}$ (see Theorem 10 of Ref. 12).

As a consequence, every state $\omega \in \mathcal{N}(\mathcal{T})_{*}$ is represented by a unique density $\sigma$ in $\mathcal{M}_{0}^{\perp}$, and in this case, we write $\omega=\omega_{\sigma}$ to mean that $\omega(x)=\operatorname{tr}(\sigma x)$ for all $x \in \mathcal{N}(\mathcal{T})$. Therefore, if we denote by $\mathcal{S}=\left(\mathcal{S}_{t}\right)_{t \geq 0}$, the restriction of $\mathcal{T}$ to $\mathcal{N}(\mathcal{T})$, we have

$$
\left(\mathcal{S}_{* t} \omega_{\sigma}\right)(x)=\omega_{\sigma}\left(\mathcal{T}_{t}(x)\right)=\operatorname{tr}\left(\sigma e^{\mathrm{i} t H} x e^{-\mathrm{i} t H}\right)=\operatorname{tr}\left(\mathcal{E}_{*}\left(e^{-\mathrm{i} t H} \sigma e^{\mathrm{i} t H}\right) x\right)
$$

for all $x=\mathcal{E}(x) \in \mathcal{N}(\mathcal{T})$, concluding that $\mathcal{S}_{* t} \omega_{\sigma}$ is represented by the density $\mathcal{E}_{*}\left(e^{-\mathrm{i} t H} \sigma e^{\mathrm{i} t H}\right) \in \mathcal{M}_{0}^{\perp}$. In an equivalent way, we have

$$
\mathcal{T}_{* t}(\sigma)=\mathcal{E}_{*}\left(e^{-\mathrm{i} t H} \sigma e^{\mathrm{i} t H}\right) \quad \forall \sigma \in \mathcal{M}_{0}^{\perp} .
$$

Theorem 15. If $\mathcal{N}(\mathcal{T})$ is atomic and there exists a faithful invariant state, then

$$
\mathcal{R}\left(\mathcal{T}_{*}\right)=\mathcal{M}_{0}^{\perp}=\left\{\sigma \in \mathfrak{I}(\mathrm{h}): \mathcal{T}_{* t}(\sigma)=\mathcal{E}_{*}\left(e^{-\mathrm{i} t H} \sigma e^{\mathrm{i} t H}\right) \forall t \geq 0\right\} \simeq \mathcal{N}(\mathcal{T})_{*}
$$

for every Hamiltonian $H$ in a GKSL representation of the generator of $\mathcal{T}$.

Proof. The inclusion $\mathcal{M}_{0}^{\perp} \subseteq\left\{\sigma \in \mathfrak{I}(\mathrm{h}): \mathcal{T}_{* t}(\sigma)=\mathcal{E}_{*}\left(e^{-\mathrm{i} t H} \sigma e^{\mathrm{i} t H}\right) \forall t \geq 0\right\}$ follows from the previous discussion. On the other hand, if we have $\mathcal{T}_{* t}(\sigma)=\mathcal{E}_{*}\left(e^{-\mathrm{i} t H} \sigma e^{\mathrm{i} t H}\right)$ for all $t \geq 0$, taking $t=0$, we get $\sigma=\mathcal{E}_{*}(\sigma)$, i.e., $\sigma$ belongs to $\mathcal{M}_{0}^{\perp}$.

Now, given $\sigma \in \mathcal{R}\left(\mathcal{T}_{*}\right)$ such that $\mathcal{T}_{* t}(\sigma)=e^{\mathrm{i} t \lambda} \sigma$ for all $t \geq 0, \lambda \in \mathbb{R}$, we have

$$
\operatorname{tr}(\sigma x)=\lim _{t \rightarrow \infty} \operatorname{tr}(\sigma x)=\lim _{t \rightarrow \infty} \operatorname{tr}\left(\mathcal{T}_{* t}(\sigma) e^{-\mathrm{i} t \lambda} x\right)=\lim _{t} e^{-\mathrm{i} t \lambda} \operatorname{tr}\left(\sigma \mathcal{T}_{t}(x)\right)=0
$$

for all $x \in \mathcal{M}_{0}$ so that $\sigma$ belongs to $\mathcal{M}_{0}^{\perp}$. This proves that $\mathcal{R}\left(\mathcal{T}_{*}\right)$ is contained in $\mathcal{M}_{0}^{\perp}$. 
In order to prove the opposite inclusion, it is enough to show that $\mathcal{N}(\mathcal{T})^{\perp}$ contains $\left.\left\{\sigma \in \mathfrak{I}(\mathrm{h}): 0 \in \overline{\mathcal{T}}_{\star t}(\sigma)\right\}_{t \geq 0}^{w}\right\}$ since we have

$$
\Im(\mathrm{h})=\mathcal{R}\left(\mathcal{T}_{*}\right) \oplus\left\{\sigma \in \mathfrak{I}(\mathrm{h}): 0 \in{\overline{\left\{\mathcal{T}_{* t}(\sigma)\right\}_{t \geq 0}}}_{t}^{w}\right\}=\mathcal{M}_{0}^{\perp} \oplus \mathcal{N}(\mathcal{T})^{\perp}
$$

by Eq. (12), item (F2), and Theorem 11. have

So, let $\sigma \in \mathfrak{I}(\mathrm{h})$ such that $0 \in{\overline{\left\{\mathcal{T}_{* t}(\sigma)\right\}_{t \geq 0}}}_{t>}^{w}$ given $\left(t_{\alpha}\right)$ with $w-\lim _{\alpha} \mathcal{T}_{* t_{\alpha}}(\sigma)=0$ and $x \in \mathfrak{M}_{r}$ be such that $\mathcal{T}_{t}(x)=e^{\mathrm{i} t \lambda} x$ for some $\lambda \in \mathbb{R}$, we

$$
\operatorname{tr}(\sigma x)=\lim _{\alpha} \operatorname{tr}\left(\sigma e^{-\mathrm{i} t \lambda} \mathcal{T}_{t_{\alpha}}(x)\right)=\lim _{\alpha} e^{-\mathrm{i} t \lambda} \operatorname{tr}\left(\mathcal{T}_{* t_{\alpha}}(\sigma) x\right)=0 .
$$

This means that $\sigma$ belongs to $\mathcal{N}(\mathcal{T})^{\perp}$ by Theorem 11 .

In general, when there does not exist a faithful invariant state, $\mathcal{R}\left(\mathcal{T}_{*}\right)$ could be different from $\mathcal{N}(\mathcal{T})_{*}$, as we can see in Example 3.

Example 3. Let us consider a generic quantum Markov semigroup with $\mathbb{C}^{3}$, more precisely the uniformly continuous QMS generated by

$$
\mathcal{L}(x)=G^{*} x+\sum_{j=1,2} L_{3 j}^{*} x L_{3 j}+x G
$$

where

$$
G=\left(-\frac{\gamma_{33}}{2}+i \kappa_{3}\right)\left|e_{3}\right\rangle\left\langle e_{3}\left|, \quad L_{3 j}=\sqrt{\gamma_{3 j}}\right| e_{j}\right\rangle\left\langle e_{3}\right| \quad \text { for } \quad j=1,2
$$

with $\kappa_{3} \in \mathbb{R}, \gamma_{3 j}>0$ for $j=1,2$, and $\gamma_{33}=-\gamma_{31}-\gamma_{32}$. We know that the restriction of $\mathcal{L}$ to the diagonal matrices is the generator of a continuous time Markov chain $\left(X_{t}\right)_{t}$ with values in $\{1,2,3\}$. For more details, see Refs. 1 and 11.

Since 1 and 2 are absorbing states for $\left(X_{t}\right)_{t}$ and 3 is a transient state, by Proposition 2 of Ref. 13, we know that any invariant state of $\mathcal{T}$ is supported on $\operatorname{span}\left\{\mathrm{e}_{1}, \mathrm{e}_{2}\right\}$. In particular, this implies that there is no faithful invariant state.

Moreover, Theorem 8 of Ref. 13 gives $\mathcal{N}(\mathcal{T})=\mathbb{C} 1$ since the absorbing states are accessible from 3. As a consequence, $\mathcal{N}(\mathcal{T})_{*}=\mathbb{C} 1$.

On the other hand, since 1 is absorbing, the state $\left|e_{1}\right\rangle\left\langle e_{1}\right|$ is invariant, and so it belongs, in particular, to $\mathcal{R}\left(\mathcal{T}_{*}\right)$. Therefore, we have $\mathcal{R}\left(\mathcal{T}_{*}\right) \neq \mathcal{N}(\mathcal{T})_{*}$.

We can now give the structure of reversible states when $\mathcal{N}(\mathcal{T})$ is atomic.

Theorem 16. Assume $\mathcal{N}(\mathcal{T})$ is atomic, and suppose there exists a faithful $\mathcal{T}$-invariant state. Let $\left(p_{i}\right)_{i \in I},\left(\mathrm{k}_{i}\right)_{i \in I}$, and $\left(\mathrm{m}_{i}\right)_{i \in I}$ be as in Theorem 5. A state $\eta$ belongs to $\mathcal{R}\left(\mathcal{T}_{*}\right)$ if and only if it can be written in the form

$$
\eta=\sum_{i \in I} \operatorname{tr}_{\mathrm{m}_{i}}\left(p_{i} \eta p_{i}\right) \otimes \tau_{\mathrm{m}_{i}}
$$

where, for every $i \in I, \tau_{\mathrm{m}_{i}}$ is the unique $\mathcal{T}^{\mathrm{m}_{i}}$-invariant state which is also faithful.

Proof. Let $\mathcal{E}$ be the conditional expectation onto $\mathcal{N}(\mathcal{T})$ given in Eq. (5), i.e.,

$$
\mathcal{E}(x)=\sum_{i}\left(\sum_{j} \mathrm{~m}_{i}\left\langle f_{j}\left|\left(\mathbb{1}_{\mathrm{k}_{i}} \otimes \tau_{\mathrm{m}_{i}}\right) x_{i i}\right| f_{j}\right\rangle_{\mathrm{m}_{i}} \otimes \mathbb{1}_{\mathrm{m}_{i}}\right)
$$

with $x_{i i}=p_{i} x p_{i}$ for all $x \in \mathcal{B}(\mathrm{h}), \tau_{\mathrm{m}_{i}}$ be the unique faithful invariant state for $\mathcal{T}^{\mathrm{m}_{i}}$, and $\left(f_{j}\right)_{j}$ be an orthonormal basis of $\mathrm{m}_{i}$ diagonalizing it.

Since $\mathcal{E}_{*}: \mathfrak{I}(\mathrm{h}) \rightarrow \mathcal{N}(\mathcal{T})_{*}=\mathcal{R}\left(\mathcal{T}_{*}\right)$, we can characterize the reversible states as the image of $\mathcal{E}_{*}$. In particular, for all $\eta \in \mathfrak{I}(\mathrm{h})$ and $x \in \mathcal{B}(\mathrm{h})$, we have

$$
\begin{aligned}
\operatorname{tr}(\eta \mathcal{E}(x)) & =\sum_{i \in I} \sum_{j} \operatorname{tr}\left(p_{i} \eta p_{i}\left(\mathrm{~m}_{i}\left\langle f_{j}\left|\left(\mathbb{1}_{\mathrm{k}_{i}} \otimes \tau_{i}\right) x_{i i}\right| f_{j}\right\rangle_{\mathrm{m}_{i}} \otimes \mathbb{1}_{\mathrm{m}_{i}}\right)\right) \\
& =\sum_{i} \sum_{j} \operatorname{tr}\left(\operatorname{tr}_{\mathrm{m}_{i}}\left(p_{i} \eta p_{i}\right)\left(\mathrm{m}_{i}\left\langle f_{j}\left|\left(\mathbb{1}_{\mathrm{k}_{i}} \otimes \tau_{i}\right) x_{i i}\right| f_{j}\right\rangle_{\mathrm{m}_{i}}\right)\right)
\end{aligned}
$$

Now, an easy computation shows that 


$$
\mathrm{m}_{i}\left\langle f_{j}\left|\left(\mathbb{1}_{\mathrm{k}_{i}} \otimes \tau_{i}\right) y\right| f_{j}\right\rangle \mathrm{m}_{i}=\sum_{l}\left\langle f_{j}, \tau_{i} f_{j}\right\rangle y_{l j}
$$

for $y=\sum_{l, k} y_{l k}\left|f_{l}\right\rangle f_{j} \mid \in \mathcal{B}\left(\mathrm{k}_{i} \otimes \mathrm{m}_{i}\right)$ so that

$$
\operatorname{tr}(\eta \mathcal{E}(x))=\sum_{i} \operatorname{tr}\left(\left(\operatorname{tr}_{\mathrm{m}_{i}}\left(p_{i} \eta p_{i}\right) \otimes \tau_{i}\right) x_{i i}\right) .
$$

We can then conclude that $\mathcal{E}_{*}(\eta)=\sum_{i} \operatorname{tr}_{\mathrm{m}_{i}}\left(p_{i} \eta p_{i}\right) \otimes \tau_{i}$, and we are done.

We have thus derived the general form of reversible states starting from the structure of the atomic decoherence-free algebra $\mathcal{N}(\mathcal{T})$. This is a well known fact for a completely positive and unitary map (i.e., a channel) on a finite-dimensional space (see, e.g., Theorem 6.16 of Ref. 32 and section $V$ of Ref. 9), but the proof of this result is not generalizable to the infinite dimensional case since it is based on a spectral decomposition of the channel.

\section{RELATIONSHIPS WITH THE STRUCTURE OF FIXED POINTS}

In this section, we investigate the structure of the set $\mathcal{F}(\mathcal{T})$ of fixed points of the semigroup and its relationships with the decomposition of $\mathcal{N}(\mathcal{T})$ given in Sec. II.

First of all, we prove the atomicity of $\mathcal{F}(\mathcal{T})$ and relate this algebra with the space of invariant states. The reader can find the proof of these results in Ref. 19, but we report them here for the sake of completeness.

Proposition 17. If there exists a faithful normal invariant state, then $\mathcal{F}(\mathcal{T})$ is an atomic algebra and $\mathcal{F}(\mathcal{T}) *$ is isomorphic to the space $\mathcal{F}\left(\mathcal{T}_{*}\right)$ of linear normal invariant functionals.

Proof. Since there exists a faithful invariant state, by Theorem 2.1 of Ref. 19 and by Ref. 20, $\mathcal{F}(\mathcal{T})$ is the image of a normal conditional expectation $\mathcal{E}: \mathcal{B}(\mathrm{h}) \rightarrow \mathcal{F}(\mathcal{T})$ given by

$$
\mathcal{E}(x)=w^{*}-\lim _{\lambda \rightarrow 0} \lambda \int_{0}^{\infty} e^{-\lambda t} \mathcal{T}_{t}(x) d t=w^{*}-\lim _{t \rightarrow+\infty} \frac{1}{t} \int_{0}^{t} \mathcal{T}_{s}(x) d s .
$$

Hence, $\mathcal{F}(\mathcal{T})$ is atomic by Theorem 6, the range of the predual operator $\mathcal{E}_{*}$ coincides with $\operatorname{Ker} \mathcal{E}^{\perp}$, and it is isomorphic to $\mathcal{F}(\mathcal{T}) *$ through the map

$$
\operatorname{Ran} \mathcal{E}_{*}=\operatorname{Ker}^{\perp} \ni \sigma \mapsto \sigma \circ \mathcal{E} \in \mathcal{F}(\mathcal{T})_{*} .
$$

Moreover, we clearly have $\operatorname{Ran} \mathcal{E}_{*}=\mathcal{F}\left(\mathcal{T}_{*}\right)$ (see also Corollary 2.2 of Ref. 19).

Therefore, assuming the existence of a faithful invariant state, we can find a countable set $J$, and two sequences $\left(\mathrm{s}_{j}\right)_{j \in J}$ and $\left(\mathrm{f}_{j}\right)_{j \in J}$ of separable Hilbert spaces such that

$$
\begin{array}{ll}
\mathrm{h} \simeq \oplus_{j \in J}\left(\mathrm{~s}_{j} \otimes \mathrm{f}_{j}\right) & \text { (unitary equivalence) }, \\
\mathcal{F}(\mathcal{T}) \simeq \oplus_{j \in J}\left(\mathcal{B}\left(\mathrm{s}_{j}\right) \otimes \mathbb{1}_{\mathrm{f}}\right) \quad(* \text {-isomorphism isometric }),
\end{array}
$$

where $\mathbb{1}_{f_{j}}$ denote the identity operator on $f_{j}$.

Even if the decomposition (16) is given up to an isometric isomorphism, for the sake of simplicity, we will identify $h$ with $\oplus_{j \epsilon J}\left(\mathrm{~s}_{j} \otimes \mathrm{f}_{j}\right)$ and $\mathcal{F}(\mathcal{T})$ with $\oplus_{j \in J}\left(\mathcal{B}\left(\mathrm{s}_{j}\right) \otimes \mathbb{1}_{\mathrm{f}_{j}}\right)$.

Now, we can state for $\mathcal{F}(\mathcal{T})$ a similar result to Theorem 5 . Note that it has already been proved for a quantum channel on a matrix algebra in Lemma 6 of Ref. 9 and in Theorems 6.12 and 6.14 of Ref. 32. Here, we extend this in the infinite-dimensional framework.

Theorem 18. Assume there exists a faithful normal invariant state. Let $\left(\mathrm{s}_{j}\right)_{j \in J}$ and $\left(\mathrm{f}_{j}\right)_{j \in J}$ be two countable sequences of Hilbert spaces such that (15) and (16) hold. Then, we have the following facts:

1. for every GKSL representation (1) of the generator $\mathcal{L}$ by means of operators $L_{\ell}, H$, we have

$$
\begin{aligned}
L_{\ell} & =\oplus_{j \in J}\left(\mathbb{1}_{\mathrm{s}_{j}} \otimes N_{\ell}^{(j)}\right) \quad \forall \ell \geq 1, \\
H & =\oplus_{j \in J}\left(\lambda_{j} \mathbb{1}_{\mathbf{s}_{j}} \otimes \mathbb{1}_{\mathrm{f}_{j}}+\mathbb{1}_{\mathrm{s}_{j}} \otimes N_{0}^{(j)}\right),
\end{aligned}
$$


where $N_{\ell}^{(j)}$ are operators on $f_{j}$ such that the series $\sum_{\ell}\left(N_{\ell}^{(j)}\right)^{*} N_{\ell}^{(j)}$ are strongly convergent for all $j \in J,\left(\lambda_{j}\right)_{j \in J}$ is a sequence of real numbers, and every $M_{0}^{(j)}$ is a self-adjoint operator on $\mathrm{f}_{\mathrm{j}}$;

2. $\mathcal{T}_{t}(x \otimes y)=x \otimes \mathcal{T}_{t}^{\mathrm{f}_{j}}(y)$ for all $x \in \mathcal{B}\left(\mathrm{s}_{j}\right)$ and $y \in \mathcal{B}\left(\mathrm{f}_{j}\right)$, for $j \in J$, where $\mathcal{T}^{\mathrm{f}_{j}}$ is the $\mathrm{QMS}$ on $\mathcal{B}\left(\mathrm{f}_{j}\right)$ generated by $\mathcal{L}^{\mathrm{f}_{j}}$, whose GKSL representation is given by $\left\{N_{\ell}^{(j)}, N_{0}^{(j)}: \ell \geq 1\right\}$;

3. every $\mathcal{T}^{\mathrm{f}_{j}}$ is irreducible and possesses a unique (faithful) normal invariant state $\tau_{\mathrm{f}_{j}}$;

4. every invariant state $\eta$ has the form $\eta=\sum_{j \in J} \sigma_{j} \otimes \tau_{\mathfrak{f}_{j}}$ with $\sigma_{j}$ being an arbitrary positive trace-class operator on $\mathrm{s}_{j}$ such that $\sum_{j \in J} \operatorname{tr}\left(\sigma_{j}\right)=1$.

Proof. Since (16) holds, like in the Proof of Theorems 3.1 and 3.2 of Ref. 15, there exist operators $\left(N_{\ell}^{(j)}\right)_{\ell}$ on $\mathcal{B}\left(\mathrm{f}_{j}\right)$ such that $L_{\ell}=\oplus_{j \in J}\left(\mathbb{1}_{s_{j}} \otimes N_{\ell}^{(j)}\right)$ for all $\ell \geq 1$ and $j \in J$.

Now, if $p_{j}$ is the orthogonal projection onto $\mathrm{s}_{j} \otimes \mathrm{f}_{j}$, we have $H=\sum_{l, m} p_{l} H_{l m} p_{m}$ with $H_{l m}: \mathrm{h}_{s_{m}} \otimes \mathrm{h}_{\mathrm{f}_{m}} \rightarrow \mathrm{h}_{\mathrm{s}_{l}} \otimes \mathrm{h}_{\mathrm{f}_{l}}$ and $H_{l m}^{*}=H_{m l}$ for all $l$, $m \in J$. Since every $x=\oplus_{j \in J}\left(x_{j} \otimes \mathbb{1}_{f_{j}}\right) \in \mathcal{F}(\mathcal{T})$ commutes with $H$, we get $0=[x, H]$, i.e.,

$$
0=\sum_{j} p_{j}\left[x_{j} \otimes \mathbb{1}_{\mathrm{f}_{j}}, H_{j j}\right] p_{j}+\sum_{j \neq m} p_{j}\left(\left(x_{j} \otimes \mathbb{1}_{\mathrm{f}_{j}}\right) H_{j m}-H_{j m}\left(x_{m} \otimes \mathbb{1}_{f_{m}}\right)\right) p_{m},
$$

which implies

$$
\left[x_{j} \otimes \mathbb{1}_{\mathfrak{f}_{j}}, H_{j j}\right]=0 \quad \forall j \in J, \quad\left(x_{j} \otimes \mathbb{1}_{\mathrm{f}_{j}}\right) H_{j m}=H_{j m}\left(x_{m} \otimes \mathbb{1}_{\mathfrak{f}_{m}}\right) \quad \forall j \neq m .
$$

The first condition is equivalent to have $H_{j j}=\lambda_{j} \mathbb{1}_{\mathrm{s}_{j}} \otimes \mathbb{1}_{\mathrm{f}_{j}}+\mathbb{1}_{\mathrm{s}_{j}} \otimes N_{0}^{(j)}$ for some $N_{0}^{(j)} \in \mathcal{B}\left(\mathrm{f}_{j}\right)$ and $\lambda_{j} \in \mathbb{R}$; the second one gives $H_{j m}=0$ for all $j \neq m$, and so we obtain item 1 .

Item 2 trivially follows. The proofs of items 3 and 4 are similar to the ones of Theorems 4.1 and 4.3, respectively, of Ref. 15.

We now want to understand the relationships between decompositions (3) and (16) making use of the notations introduced in Theorems 5 and 18. In particular, in Theorem 19, we find a spectral characterization of the decomposition of the fixed point algebra up to an isometric isomorphism. Indeed, in this representation, the spaces $\mathrm{s}_{j}$ undergoing trivial evolutions are the eigenspaces of suitable Hamiltonians $K_{i}$ corresponding to their different eigenvalues.

First of all, we introduce the following notation: for every $i \in I$ denoted by

$$
\sigma\left(K_{i}\right):=\left\{\kappa_{j}^{(i)}: j \in J_{i}\right\}
$$

with $\kappa_{j}^{(i)} \neq \kappa_{l}^{(i)}$ for $j \neq l$ in $J_{i}$, the (pure point) spectrum of the Hamiltonian $K_{i} \in \mathcal{B}\left(\mathrm{k}_{i}\right)$ for some at most countable set $J_{i} \subseteq \mathbb{N}$. Note that if $\mathcal{T}$ has a faithful normal invariant state, then $\sigma\left(K_{i}\right)$ is exactly the spectrum of $K_{i}$ thanks to Theorem 5 .

Without loss of generality, we can choose the family $\left\{J_{i}: i \in I\right\}$ such that $J_{h} \cap J_{l}=\emptyset$ whenever $h \neq l$. In this way, set

$$
J:=\cup_{i \in I} J_{i},
$$

for $j \in J$ there exists a unique $i \in I$ such that $j=j_{i} \in J_{i}$.

Theorem 19. Assume $\mathcal{N}(\mathcal{T})$ is atomic, and let $\mathcal{N}(\mathcal{T})=\oplus_{i \in I}\left(\mathcal{B}\left(\mathrm{k}_{i}\right) \otimes \mathbb{1}_{\mathrm{m}_{i}}\right)$ with $\left(\mathrm{k}_{i}\right)_{i}$ and $\left(\mathrm{m}_{i}\right)_{i}$ being two countable sequences of Hilbert spaces such that $h=\oplus_{i \in I}\left(\mathrm{k}_{\mathrm{i}} \otimes \mathrm{m}_{\mathrm{i}}\right)$. If there exists a faithful normal invariant state, up to an isometric isomorphism, we have

$$
\mathcal{F}(\mathcal{T})=\oplus_{j \in J}\left(\mathcal{B}\left(\mathrm{s}_{j}\right) \otimes \mathbb{1}_{\mathrm{f}_{j}}\right)
$$

with $J$ defined in (18), and

$$
\mathrm{s}_{j}=\mathrm{s}_{j_{i}}:=\operatorname{Ker}\left(K_{i}-\kappa_{j}^{(i)} \mathbb{1}_{\mathrm{k}_{i}}\right), \quad \mathrm{f}_{j}=\mathrm{f}_{j_{i}}:=\mathrm{m}_{i} \quad \forall j_{i} \in J_{i}, i \in I .
$$

Proof. By considering the spectral decomposition $K_{i}=\sum_{j \in J_{i}} k_{j}^{(i)} q_{j i}$ with $\left(q_{j i}\right)_{j \in J_{i}}$ mutually orthogonal projections such that

$$
q_{j i} k_{i}=\operatorname{Ker}\left(K_{i}-\kappa_{j}^{(i)} \mathbb{1}_{k_{i}}\right)=: \mathrm{s}_{j_{i}}
$$

and $\sum_{j \in J_{i}} q_{j i}=\mathbb{1}_{m_{i}}$, we immediately obtain 


$$
\mathrm{k}_{i} \otimes \mathrm{m}_{i}=\left(\oplus_{j \in J_{i}} \mathrm{~s}_{j_{i}}\right) \otimes \mathrm{m}_{i}=\oplus_{j \in J_{i}}\left(\mathrm{~s}_{j_{i}} \otimes \mathrm{f}_{j_{i}}\right)
$$

by setting $f_{j_{i}}:=\mathrm{m}_{i}$ for all $j \in J_{i}$. Therefore, by the definition of $J$, since every $j \in J$ belongs to a unique $J_{i}$, we have

$$
\mathrm{h}=\oplus_{i \in I}\left(\mathrm{k}_{i} \otimes \mathrm{m}_{i}\right)=\oplus_{i \in I} \oplus_{j \in J_{i}}\left(\mathrm{~s}_{j_{i}} \otimes \mathrm{f}_{j_{i}}\right)=\oplus_{j \in J}\left(\mathrm{~s}_{j} \otimes \mathrm{f}_{j}\right) .
$$

In order to conclude the proof, we have to show equality (19). Given $x \in \mathcal{F}(\mathcal{T}) \subseteq \mathcal{N}(\mathcal{T})$ (see item 4 of Proposition 2), we can write $x=\oplus_{i \in I}\left(x_{i} \otimes \mathbb{1}_{\mathrm{m}_{i}}\right)$ with $\left(x_{i}\right)_{i \in I} \subseteq \mathcal{B}\left(\mathrm{k}_{i}\right)$, and so, by Theorem 5 , we have

$$
x=\oplus_{i \in I}\left(x_{i} \otimes \mathbb{1}_{\mathrm{m}_{i}}\right)=\mathcal{T}_{t}\left(\oplus_{i \in I}\left(x_{i} \otimes \mathbb{1}_{\mathrm{m}_{i}}\right)\right)=\oplus_{i \in I}\left(e^{\mathrm{i} t K_{i}} x_{i} e^{-\mathrm{i} t K_{i}} \otimes \mathbb{1}_{\mathrm{m}_{i}}\right) .
$$

Consequently, $x_{i}=e^{\mathrm{it} K_{i}} x_{i} e^{-\mathrm{i} t K_{i}}$ for all $i \in I$, i.e., every $x_{i}$ commutes with $K_{i}$, and then with each projection $q_{j i}$ with $j \in J_{i}$. This means that each $x_{i}=\oplus_{j \in \epsilon_{i}} q_{j i} x_{i} q_{j i}$ belongs to the algebra $\oplus_{j \in \epsilon_{i}} q_{j i} \mathcal{B}\left(\mathrm{k}_{i}\right) q_{j i}=\oplus_{j \in J_{i}} \mathcal{B}\left(q_{j j} \mathrm{k}_{i}\right)=\oplus_{j \in J_{i}} \mathcal{B}\left(\mathrm{s}_{j}\right)$ so that $x$ is in $\oplus_{j \in J}\left(\mathcal{B}\left(\mathrm{s}_{j}\right) \otimes \mathbb{1}_{\mathrm{f} j}\right)$.

On the other hand, given $i \in I$ and $j \in J_{i}$, for $u, v \in \mathrm{S}_{j}=\operatorname{Ker}\left(K_{i}-\kappa_{j}^{(i)} \mathbb{1}_{\mathrm{k}_{i}}\right)$, we get

$$
\mathcal{T}_{t}\left(|u\rangle\langle v| \otimes \mathbb{1}_{\mathrm{m}_{i}}\right)=\left|e^{\mathrm{i} t K_{i}} u\right\rangle\left\langle e^{\mathrm{i} t K_{i}} v\left|\otimes \mathbb{1}_{\mathrm{m}_{i}}=\right| u\right\rangle\langle v| \otimes \mathbb{1}_{\mathrm{m}_{i}} \quad \forall t \geq 0,
$$

where $u$ and $v$ are eigenvectors of $K_{i}$ associated with the same eigenvalue $\kappa_{j}^{(i)}$. Since $\operatorname{Ker}\left(K_{i}-\kappa_{j}^{(i)} \mathbb{1}_{\mathrm{k}_{i}}\right)$ is generated by elements of the form $|u\rangle\langle v|$ and the net $\left(\mathcal{T}_{t}(z)\right)_{t}$ is uniformly bounded for all $z$, we obtain the inclusion $\mathcal{B}\left(\mathrm{s}_{j}\right) \otimes \mathbb{1}_{\mathrm{m}_{i}} \subseteq \mathcal{F}(\mathcal{T})$ for all $j \in J_{i}$ and $i \in I$, i.e., $\mathcal{B}\left(\mathrm{s}_{j}\right) \otimes \mathbb{1}_{\mathrm{f}_{j}}$ $\subseteq \mathcal{F}(\mathcal{T})$ for all $j \in J=\cup_{i \in I} J_{i}$.

Theorem below shows how we can derive an "atomic decomposition" of $\mathcal{F}(\mathcal{T})$ from one of $\mathcal{N}(\mathcal{T})$. Moreover, it is also possible to obtain the decomposition of operators $\left\{H, L_{\ell}\right\} \ell$ according to the atomic splitting of fixed point algebra, starting from that one of $\mathcal{N}(\mathcal{T})$, i.e., $L_{\ell}=\oplus_{i \in I}\left(\mathbb{1}_{\mathrm{k}_{i}} \otimes M_{\ell}^{(i)}\right)$ and $H=\oplus_{i \in I}\left(K_{i} \otimes \mathbb{1}_{\mathrm{m}_{i}}+\mathbb{1}_{\mathrm{k}_{i}} \otimes M_{0}^{(i)}\right)$ with $K_{i} \in \mathcal{B}\left(\mathrm{k}_{i}\right)$ and $M_{0}^{(i)}, M_{\ell}^{(i)}$ in $\mathcal{B}\left(\mathrm{m}_{i}\right)$.

More precisely, given $i \in I$, since $\sum_{j \in I_{i}} q_{j i}=\mathbb{1}_{m_{i}}$, we have

$$
\mathbb{1}_{\mathrm{k}_{i}} \otimes M_{\ell}^{(i)}=\left(\oplus_{j \in I_{i}} q_{j i}\right) \otimes M_{\ell}^{(i)}=\oplus_{j \in I_{i}}\left(q_{j i} \otimes M_{\ell}^{(i)}\right)
$$

so that

$$
L_{\ell}=\oplus_{i \in I}\left(\oplus_{j \in I_{i}}\left(q_{j i} \otimes M_{\ell}^{(i)}\right)\right) .
$$

Similarly, recalling the spectral decomposition of $K_{i}, K_{i}=\sum_{j \in J_{i}} k_{j}^{(i)} q_{j i}$, we can write $H$ as

$$
H=\oplus_{i \in I}\left(\oplus_{j \in I_{i}}\left(\kappa_{j}^{(i)} q_{j i} \otimes \mathbb{1}_{m_{i}}+q_{j i} \otimes M_{0}^{(i)}\right)\right) .
$$

Finally, since $q_{j i}$ is the identity of $\mathrm{s}_{j}$ and $\mathrm{m}_{i}=\mathrm{f}_{j}$ for all $j \in I_{i}$, we get

$$
H=\oplus_{i \in I}\left(\oplus_{j \in I_{i}}\left(\kappa_{j}^{(i)} \mathbb{1}_{\mathrm{s}_{j}} \otimes \mathbb{1}_{\mathrm{f}_{i}}+\mathbb{1}_{\mathrm{s}_{j}} \otimes M_{0}^{(i)}\right)\right) .
$$

We now want to analyze the opposite procedure.

Assuming $\mathcal{F}(\mathcal{T})=\oplus_{j \in J}\left(\mathcal{B}\left(\mathrm{s}_{j}\right) \otimes \mathbb{1}_{\mathrm{f}_{j}}\right)$ with $\left(\mathrm{s}_{j}\right)_{j}$ and $\left(\mathrm{f}_{j}\right)_{j}$ being two countable sequences of Hilbert spaces such that $\mathrm{h}=\oplus_{j \in j}\left(\mathrm{~s}_{j} \otimes \mathrm{f}_{j}\right)$ and using notations of Theorem 18 , we set an equivalence relation on $J$ in the following way:

Definition 20. Given $j, k \in J$, we say that $j$ is in relation with $k$ (and write $j \sim k$ ) if there exist a complex separable Hilbert space $m$ and unitary isomorphisms

$$
V_{j}: \mathrm{f}_{j} \rightarrow \mathrm{m}, \quad V_{k}: \mathrm{f}_{k} \rightarrow \mathrm{m}
$$

such that operators $\left\{V_{j} N_{l}^{(j)} V_{j}^{*}, V_{j} N_{0}^{(j)} V_{j}^{*}: l \geq 1\right\}$ and $\left\{V_{k} N_{l}^{(k)} V_{k}^{*}, V_{k} N_{0}^{(k)} V_{k}^{*}: l \geq 1\right\}$ give the same Lindbladian operator on $\mathcal{B}(\mathrm{m})$.

We obtain in this way an equivalence relation which induces a partition of $J, J=\cup_{n \in I} I_{n}$, for some finite or countable set $I \subseteq \mathbb{N}$, where each $I_{n}$ is an equivalence class with respect to $\sim$. 
Theorem 21. Assume that there exists a faithful normal invariant state and $\mathcal{N}(\mathcal{T})$ is atomic. Let $\mathcal{F}(\mathcal{T})=\oplus_{j \in J}\left(\mathcal{B}\left(\mathrm{s}_{j}\right) \otimes \mathbb{1}_{\mathrm{f}_{j}}\right)$ with $\left(\mathrm{s}_{j}\right)_{j}$ and $\left(\mathrm{f}_{j}\right)_{j}$ being two countable sequences of Hilbert spaces such that $h=\oplus_{j \in j}\left(\mathrm{~S}_{j} \otimes f_{j}\right)$, and let $\left\{I_{n}: n \in I\right\}$ be the set of equivalence classes of $J$ with respect to the relation $\sim$. Then, $\mathcal{N}(\mathcal{T})$ is isometrically isomorphic to the direct sum $\oplus_{n \in I}\left(\mathcal{B}\left(\mathrm{k}_{n}\right) \otimes \mathbb{1}_{\mathrm{m}_{n}}\right)$ with

$$
\mathrm{k}_{n}:=\oplus_{j \in I_{n}} \mathrm{~S}_{j}, \quad \mathrm{~m}_{n}:=V_{j} \mathrm{f}_{j} \quad \forall j \in I_{n},
$$

where $V_{j}$ 's are the unitary isomorphisms given in (21).

Proof. Given $n \in I$, by definition of $\mathrm{m}_{n}$, we can define a unitary operator by setting

$$
\begin{aligned}
U_{n}: \oplus_{j \in I_{n}}\left(\mathrm{~s}_{j} \otimes \mathrm{f}_{j}\right) & \rightarrow\left(\oplus_{j \in I_{n}} \mathrm{~s}_{j}\right) \otimes \mathrm{m}_{n}=\mathrm{k}_{n} \otimes \mathrm{m}_{n}, \\
\oplus_{j \in I_{n}}\left(u_{j} \otimes z_{j}\right) & \mapsto \sum_{j \in I_{n}}\left(u_{j}^{(j)} \otimes V_{j} z_{l}\right),
\end{aligned}
$$

where $u_{j}^{(j)}$ in $\oplus_{i \in I_{n}} \mathrm{~h}_{s_{i}}$ denotes the vector

$$
u_{j}^{(j)}:=\oplus_{i \in I_{n}} v_{i}, \quad v_{i}:= \begin{cases}0 & \text { if } i \neq j \\ u_{j} & \text { if } i=j .\end{cases}
$$

Now, since

$$
\mathrm{h}=\oplus_{j \in j}\left(\mathrm{~s}_{j} \otimes \mathrm{f}_{j}\right)=\oplus_{n \in I}\left(\oplus_{j \in I_{n}}\left(\mathrm{~s}_{j} \otimes \mathrm{f}_{j}\right)\right)
$$

by the equality $J=\cup_{n \in I} I_{n}$, by setting $U:=\oplus_{n \in I} U_{n}$, we get a unitary operator $U: \mathrm{h} \rightarrow \oplus_{n \in I}\left(\mathrm{k}_{n} \otimes \mathrm{m}_{n}\right)$ such that

$$
U \mathcal{F}(\mathcal{T}) U^{*}=\oplus_{n \in I}\left(\left(\oplus_{j \in I_{n}} \mathcal{B}\left(\mathrm{s}_{j}\right)\right) \otimes \mathbb{1}_{\mathrm{m}_{n}}\right) .
$$

In order to conclude the proof, we have to show that

$$
U \mathcal{N}(\mathcal{T}) U^{*}=\oplus_{n \in I}\left(\mathcal{B}\left(\oplus_{j \in I_{n}} \mathrm{~s}_{j}\right) \otimes \mathbb{1}_{\mathrm{m}_{n}}\right) .
$$

To this end, recall that by Theorem 18 , the operators $\left(L_{\ell}\right)_{\ell}, H$ in a GKSL representation of the generator $\mathcal{L}$ can be written as

$$
L_{\ell}=\oplus_{j \in J}\left(\mathbb{1}_{\mathrm{s}_{j}} \otimes N_{\ell}^{(j)}\right) \quad \forall \ell \geq 1, \quad H=\oplus_{j \in J}\left(\lambda_{j} \mathbb{1}_{\mathrm{s}_{j}} \otimes \mathbb{1}_{\mathrm{f}_{j}}+\mathbb{1}_{\mathrm{s}_{j}} \otimes N_{0}^{(j)}\right)
$$

with $\left(N_{\ell}^{(j)}\right)_{\ell \geq 1} \subseteq \mathcal{B}\left(\mathrm{s}_{j}\right), N_{0}^{(j)}=\left(N_{0}^{(j)}\right)^{*} \in \mathcal{B}\left(\mathrm{f}_{j}\right)$, and $\lambda_{j} \in \mathbb{R}$ for all $j \in J$; moreover, by the definition of $I_{n}$, we can choose operators $\left(M_{l}^{(n)}\right)_{l}$ and $M_{0}^{(n)}=\left(M_{0}^{(n)}\right)^{*}$ in $\mathcal{B}\left(\mathrm{m}_{n}\right)$ such that $\left\{M_{l}^{(n)}, M_{0}^{(n)}: l \geq 1\right\}$ is a GKSL representation of the generator $\mathcal{L}^{\mathrm{m}_{n}}$ of a QMS $\mathcal{T}^{\mathrm{m}_{n}}$ on $\mathcal{B}\left(\mathrm{m}_{n}\right)$ equivalent to $\left\{V_{j} N_{l}^{(j)} V_{j}^{*}, V_{j} N_{0}^{(j)} V_{j}^{*}: l \geq 1\right\}$ for all $j \in I_{n}$. Therefore, the operators

$$
\begin{aligned}
L_{\ell}^{\prime} & :=\oplus_{n \in I}\left(\oplus_{j \in I_{n}}\left(\mathbb{1}_{s_{j}} \otimes V_{j}^{*} M_{\ell}^{(n)} V_{j}\right)\right), \\
H^{\prime} & :=\oplus_{n \in I}\left(\oplus_{j \in I_{n}}\left(\lambda_{j} \mathbb{1}_{s_{j}} \otimes \mathbb{1}_{\mathrm{f}_{j}}\right)+\oplus_{j \in I_{n}}\left(\mathbb{1}_{\mathrm{s}_{j}} \otimes V_{j}^{*} M_{0}^{(n)} V_{j}\right)\right)
\end{aligned}
$$

clearly give the same GKSL representation of $\left\{L_{\ell}, H: \ell \geq 1\right\}$. Moreover, we have

$$
\begin{aligned}
U L_{\ell}^{\prime} U^{*} & =\oplus_{n \in I} U_{n}\left(\oplus_{j \in I_{n}}\left(\mathbb{1}_{\mathrm{s}_{j}} \otimes V_{j}^{*} M_{\ell}^{(n)} V_{j}\right)\right) U_{n}^{*}=\oplus_{n \in I}\left(\mathbb{1}_{\mathrm{k}_{n}} \otimes M_{\ell}^{(n)}\right), \\
U H^{\prime} U^{*} & =\oplus_{n \in I} U_{n}\left(\oplus_{j \in I_{n}}\left(\lambda_{j} \mathbb{1}_{\mathrm{s}_{j}} \otimes \mathbb{1}_{\mathrm{f}_{\mathrm{j}}}\right)+\oplus_{j \in I_{n}}\left(\mathbb{1}_{\mathrm{s}_{j}} \otimes V_{j}^{*} M_{0}^{(n)} V_{j}\right)\right) U_{n}^{*} \\
& =\oplus_{n \in I}\left(K_{n} \otimes \mathbb{1}_{\mathrm{m}_{n}}+\mathbb{1}_{\mathrm{k}_{n}} \otimes M_{0}^{(n)}\right)
\end{aligned}
$$

with $K_{n}:=\left(\oplus_{j \in I_{n}} \lambda_{j} \mathbb{1}_{\mathrm{s}_{j}}\right)=K_{n}^{*} \in \mathcal{B}\left(\mathrm{k}_{n}\right)$ so that

$$
\begin{gathered}
U \delta_{H \prime}^{m}\left(L_{\ell}^{\prime}\right) U^{*}=\delta_{U H^{\prime} U^{*}}^{m}\left(U L_{\ell}^{\prime} U^{*}\right)=\oplus_{n \in I}\left(\mathbb{1}_{\mathrm{k}_{n}} \otimes \delta_{M_{0}^{(n)}}^{m}\left(M_{\ell}^{(n)}\right)\right), \\
U \delta_{H \prime}^{m}\left(L_{\ell}^{\prime *}\right) U^{*}=\delta_{U H^{\prime} U^{*}}^{m}\left(U L_{\ell}^{\prime *} U^{*}\right)=\oplus_{n \in I}\left(\mathbb{1}_{\mathrm{k}_{n}} \otimes \delta_{M_{0}^{(n)}}^{m}\left(M_{\ell}^{(n) *}\right)\right)
\end{gathered}
$$

for all $m \geq 0$. Therefore, since $U \mathcal{N}(\mathcal{T}) U^{*}=\left\{U \delta_{H \prime}^{m}\left(L_{\ell}^{\prime}\right) U^{*}, U \delta_{H \prime}^{m}\left(L_{\ell}^{\prime *}\right) U^{*}: m \geq 0\right\}^{\prime}$ by item 2 of Proposition 2, we obtain that an operator $x \in \mathcal{B}\left(\oplus_{n \in I}\left(\mathrm{k}_{n} \otimes \mathrm{m}_{n}\right)\right)$ belongs to $U \mathcal{N}(\mathcal{T}) U^{*}$ if and only if it commutes with 


$$
\oplus_{n \in I}\left(\mathbb{1}_{\mathfrak{k}_{n}} \otimes \delta_{M_{0}^{(n)}}^{m}\left(M_{\ell}^{(n)}\right)\right), \quad \oplus_{n \in I}\left(\mathbb{1}_{\mathfrak{k}_{n}} \otimes \delta_{M_{0}^{(n)}}^{m}\left(M_{\ell}^{(n) *}\right)\right)
$$

for every $m \geq 0$.

Now, let $q_{n}:=\mathbb{1}_{\mathrm{k}_{n}} \otimes \mathbb{1}_{\mathrm{m}_{n}}$ be the orthogonal projection onto $\mathrm{k}_{n} \otimes \mathrm{m}_{n}$. We clearly have $q_{n} \in U \mathcal{F}(\mathcal{T}) U^{*}$ and $\sum_{n \in I}{ }^{q n}=\mathbb{1}$. Therefore, the algebra $q_{n} \mathcal{B}(\mathrm{h}) q_{n}=\mathcal{B}\left(\mathrm{k}_{n} \otimes \mathrm{m}_{n}\right)$ is preserved by the action of every map $\widetilde{\mathcal{T}}_{t}:=U \mathcal{T}_{t}\left(U^{*} \cdot U\right) U^{*}$, and so we can consider the restriction of $\widetilde{\mathcal{T}}$ to this algebra, getting a QMS on $\mathcal{B}\left(\mathrm{k}_{n} \otimes \mathrm{m}_{n}\right)$ denoted by $\mathcal{T}^{(n)}$ and satisfying $\mathcal{N}\left(\mathcal{T}^{(n)}\right)=q_{n} U \mathcal{N}(\mathcal{T}) U^{*} q_{n}$, where $\mathcal{N}\left(\mathcal{T}^{(n)}\right)$ is the decoherencefree algebra of $\mathcal{T}^{(n)}$. Note that since each $q_{n}$ commutes with every $U L_{\ell}{ }^{\prime} U^{*}, U L_{\ell}^{\prime *} U^{*}$, and $U H^{\prime} U^{*}$, a GKSL representation of the generator $\mathcal{L}^{(n)}$ of $\mathcal{T}^{(n)}$ is given by operators

$$
q_{n} U H^{\prime} U^{*} q_{n}=K_{n} \otimes \mathbb{1}_{\mathrm{m}_{n}}+\mathbb{1}_{\mathrm{k}_{n}} \otimes M_{0}^{(n)}, \quad q_{n} U L_{\ell}^{\prime} U^{*} q_{n}=\mathbb{1}_{\mathrm{k}_{n}} \otimes M_{\ell}^{(n)}
$$

so that

$$
\widetilde{\mathcal{T}}_{t}(x \otimes y)=e^{\mathrm{i} t K_{n}} x e^{-\mathrm{i} t K_{n}} \otimes \mathcal{T}_{t}^{\mathrm{m}_{n}}(y) \quad \forall x \in \mathcal{B}\left(\mathrm{k}_{n}\right), y \in \mathcal{B}\left(\mathrm{m}_{n}\right)
$$

and

$$
\begin{aligned}
\mathcal{N}\left(\mathcal{T}^{(n)}\right) & =\left\{\mathbb{1}_{\mathrm{k}_{n}} \otimes \delta_{M_{0}^{(n)}}^{m}\left(M_{\ell}^{(n)}\right), \mathbb{1}_{\mathrm{k}_{n}} \otimes \delta_{M_{0}^{(n)}}^{m}\left(M_{\ell}^{(n) *}\right): \ell \geq 1, m \geq 0\right\}^{\prime} \\
& =\mathcal{B}\left(\mathrm{k}_{n}\right) \otimes \mathcal{N}\left(\mathcal{T}^{\mathrm{m}_{n}}\right) .
\end{aligned}
$$

Since

$$
U \mathcal{N}(\mathcal{T}) U^{*}=\bigoplus_{n, m \in I} q_{n} U \mathcal{N}(\mathcal{T}) U^{*} q_{m}
$$

and Eq. (23) holds, to conclude the proof, we have to show that

$$
q_{n} U \mathcal{N}(\mathcal{T}) U^{*} q_{m}=\{0\} \quad \forall n \neq m \quad \text { and } \quad \mathcal{N}\left(\mathcal{T}^{\mathrm{m}_{n}}\right)=\mathbb{C} \mathbb{1}_{\mathrm{m}_{n}} .
$$

So, let $x \in U \mathcal{N}(\mathcal{T}) U^{*}$ and consider $n, m \in I$ with $n \neq m$. Since the net $\left(\mathcal{T}_{t}\left(q_{n} x q_{m}\right)\right)_{t \geq 0}$ is bound in the norm and the unit ball is weakly ${ }^{*}$ compact, there exists a weak ${ }^{*}$ cluster point $y$ such that $y=w^{*}-\lim _{\alpha} \mathcal{T}_{t_{\alpha}}\left(q_{n} x q_{m}\right)$. Therefore, for $\sigma \in \mathcal{R}\left(\mathcal{T}_{*}\right)$ with $\mathcal{T}_{* t}(\sigma)=e^{\mathrm{i} t \lambda} \sigma$ for some $\lambda \in \mathbb{R}$, we have

$$
\operatorname{tr}(\sigma y)=\lim _{\alpha} \operatorname{tr}\left(\sigma \mathcal{T}_{t_{\alpha}}\left(q_{n} x q_{m}\right)\right)=\lim _{\alpha} e^{\mathrm{i} t_{\alpha} \lambda} \operatorname{tr}\left(\sigma q_{n} x q_{m}\right) .
$$

Now, if $\lambda \neq 0$, this implies $\operatorname{tr}\left(\sigma q_{n} x q_{m}\right)=0=\operatorname{tr}(\sigma y)$; otherwise, since $\sigma$ is an invariant state, we automatically have $q_{m} \sigma q_{n}=0$ for $n \neq m$ by Theorem 18 so that $\operatorname{tr}(\sigma y)$ is 0 again. Consequently, $\operatorname{tr}(\sigma y)=0$ for all $\sigma \in \mathcal{R}\left(\mathcal{T}_{*}\right)$. On the other hand, taking $\eta$ such that $\left.0 \in \overline{\mathcal{T}}_{* t}(\eta)\right\}_{t \geq 0}^{w}$, up to passing to generalized subsequences, we have

$$
\operatorname{tr}(\eta y)=\lim _{\alpha} \operatorname{tr}\left(\eta \mathcal{T}_{t_{\alpha}}\left(q_{n} x q_{m}\right)\right)=\lim _{\alpha} \operatorname{tr}\left(\mathcal{T}_{* t_{\alpha}}(\eta) q_{n} x q_{m}\right)=0 .
$$

We can then conclude that $\operatorname{tr}(\sigma y)=0$ for all $\sigma \in \Im\left(\oplus_{n}\left(\mathrm{k}_{n} \otimes \mathrm{m}_{n}\right)\right)$ by virtue of Eq. (12), and so $y=0$. This means that $q_{n} x q_{m}$ belongs to $\mathcal{N}(\mathcal{T}) \cap \mathfrak{M}_{s}$ and this intersection is $\{0\}$ by Corollary $11\left(\mathcal{N}(\mathcal{T})\right.$ is atomic). Therefore, $q_{n} x q_{m}=0$ for $n \neq m$ and so

$$
U \mathcal{N}(\mathcal{T}) U^{*}=\oplus_{n \in I} q_{n} \mathcal{N}(\mathcal{T}) q_{n}=\oplus_{n \in I} \mathcal{N}\left(\mathcal{T}^{(n)}\right) .
$$

Moreover, since $\mathcal{N}(\mathcal{T})$ is atomic by Corollary 11, the general theory of von Neumann algebras (see, e.g., Ref. 29) says that each algebra $\mathcal{N}\left(\mathcal{T}^{(n)}\right)=\mathcal{B}\left(\mathrm{k}_{n}\right) \otimes \mathcal{N}\left(\mathcal{T}^{\mathrm{m}_{n}}\right)$ is atomic too, and consequently, $\mathcal{N}\left(\mathcal{T}^{\mathrm{m}_{n}}\right)$ is atomic. Finally, recalling that by construction, $\mathcal{T}_{t}^{\mathrm{m}_{n}}=\mathcal{T}_{t}^{\mathrm{f}_{j}}$ for all $j \in I_{n}$ with $\mathcal{T}_{t}^{f_{j}}$ being an irreducible QMS having a faithful invariant state (see Theorem 18), we get $\mathcal{N}\left(\mathcal{T}^{\mathrm{m}_{n}}\right)=\mathbb{C} \mathbb{1}_{m_{n}}$ thanks to Proposition 4.3 of Ref. 15.

Remark 22. Theorem 21 provides, in particular, a way to go from the decomposition of GKSL operators $H,\left(L_{l}\right)_{l}$ of $\mathcal{L}$ according to the splitting of $h=\oplus_{j \in j}\left(s_{j} \otimes f_{j}\right)$ associated with the atomic algebra $\mathcal{F}(\mathcal{T})$, to the other one decomposition with respect to the splitting $\mathrm{h}=\oplus_{n \in I}\left(\mathrm{k}_{n} \otimes \mathrm{m}_{n}\right)$ associated with $\mathcal{N}(\mathcal{T})$.

More precisely, if $\mathcal{F}(\mathcal{T})=\oplus_{j \in J}\left(\mathcal{B}\left(\mathrm{s}_{j}\right) \otimes \mathbb{1}_{\mathrm{f}_{j}}\right)$ and

$$
L_{\ell}=\oplus_{j \in J}\left(\mathbb{1}_{\mathrm{s}_{j}} \otimes N_{\ell}^{(j)}\right) \quad \forall \ell \geq 1, \quad H=\oplus_{j \in J}\left(\lambda_{j} \mathbb{1}_{\mathrm{s}_{j}} \otimes \mathbb{1}_{\mathrm{f}_{j}}+\mathbb{1}_{\mathrm{s}_{j}} \otimes N_{0}^{(j)}\right)
$$


with $\left(N_{\ell}^{(j)}\right)_{\ell \geq 1} \subseteq \mathcal{B}\left(\mathrm{s}_{j}\right), N_{0}^{(j)} \in \mathcal{B}\left(\mathrm{f}_{j}\right)$, and $\lambda_{j} \in \mathbb{R}$ for all $j \in J$, then we can decompose $H,\left(L_{\ell}\right)_{\ell}$ with respect to the splitting $\mathrm{h}=\oplus_{n \in I}\left(\mathrm{k}_{n} \otimes \mathrm{m}_{n}\right)$ given by (22) as follows:

$$
L_{\ell}=\oplus_{n \in I}\left(\mathbb{1}_{\mathrm{k}_{n}} \otimes M_{\ell}^{(n)}\right), \quad H=\oplus_{n \in I}\left(K_{n} \otimes \mathbb{1}_{\mathrm{m}_{n}}+\mathbb{1}_{\mathrm{k}_{n}} \otimes M_{0}^{(n)}\right),
$$

where $\left(M_{\ell}^{(n)}\right)_{\ell \geq 1}, M_{0}^{(n)}=M_{0}^{(n) *}$ are operators in $\mathcal{B}\left(\mathrm{m}_{n}\right)$ giving the same GKSL representation of $\left(N_{l}^{(j)}\right)_{\ell \geq 1}, N_{0}^{(j)}$ for all $j \in I_{n}$ and every $K_{n}$ is the self-adjoint operator on $\mathcal{B}\left(\mathrm{K}_{n}\right)$ having $\left(\lambda_{j}\right)_{j \in I_{n}}$ as eigenvalues and $\left(\mathrm{s}_{j}\right)_{j \in I_{n}}$ as corresponding eigenspaces.

\section{ACKNOWLEDGMENTS}

The financial support of MIUR FIRB 2010 Project No. RBFR10COAQ "Quantum Markov Semigroups and their Empirical Estimation" is gratefully acknowledged.

\section{REFERENCES}

${ }^{1}$ L. Accardi, F. Fagnola, and S. Hachicha, "Generic q-Markov semigroups and speed of convergence of q-algortithms," Infinite Dimens. Anal. Quantum Probab. Relat. Top. 9,567 (2006).

${ }^{2}$ J. Agredo, F. Fagnola, and R. Rebolledo, “Decoherence free subspaces of a quantum Markov semigroup,” J. Math. Phys. 55, 112201 (2014).

${ }^{3}$ V. V. Albert, B. Bradlyn, M. Fraas, and L. Jiang, "Geometry and response of Lindbladians," Phys. Rev. X 6, 041031 (2016).

${ }^{4}$ S. Albeverio and R. Høegh-Krohn, "Frobenius theory for positive maps of von Neumann algebras," Commun. Math. Phys. 64, 83-94 (1978).

${ }^{5}$ A. Batkai, U. Groh, D. Kunszenti-Kovacs, and M. Schreiber, “Decomposition of operator semigroups on $W^{*}$-algebras," Semigroup Forum 84(1), 8-24 (2012).

${ }^{6}$ B. Baumgartner and H. Narnhofer, "The structure of state space concerning quantum dynamical semigroups," Rev. Math. Phys. 24(2), 1250001 (2012).

${ }^{7}$ B. Blackadar, "Operator algebra," in Encyclopaedia of Mathematical Sciences (Springer, New York, 2006), Vol. 122.

${ }^{8} \mathrm{Ph}$. Blanchard and R. Olkiewicz, "Decoherence induced transition form quantum to classical dynamics," Rev. Math. Phys. 15(3), 217-243 (2003).

${ }^{9}$ R. Blume-Kohout, H. K. Ng, D. Poulin, and L. Viola, “Information-preserving structures: A general framework for quantum zero-error information," Phys. Rev. A 82, 062306 (2010).

${ }^{10}$ R. Carbone, E. Sasso, and V. Umanità, “Decoherence for quantum Markov semigroups on matrix spaces,” Ann. Henri Poincaré 14(4), 681-697 (2013).

${ }^{11}$ R. Carbone, E. Sasso, and V. Umanità, “On the asymptotic behavior of generic quantum Markov semigroups," Infin. Dimens. Anal. Quantum Probab. Relat. Top. 17(1), 1450001 (2014).

${ }^{12}$ R. Carbone, E. Sasso, and V. Umanità, “Environment induced decoherence for Markovian evolutions,” J. Math. Phys. 56, 092704 (2015).

${ }^{13}$ R. Carbone, E. Sasso, and V. Umanità, “Some remarks on decoherence for generic quantum Markov semigroup," Infin. Dimens. Anal. Quantum Probab. Relat. Top. 20, $1750012(2017)$.

${ }^{14}$ E. Christensen and E. D. Evans, “Cohomology of operator algebras and quantum dynamical semigroups,” J. London Math. Soc. s2-20(2), 358-368 (1979).

${ }^{15}$ J. Deschamps, F. Fagnola, E. Sasso, and V. Umanità, "Structure of uniformly continuous quantum Markov semigroups,” Rev. Math. Phys. 28(1), 165003 (2016).

${ }^{16}$ A. Dhahri, F. Fagnola, and R. Rebolledo, “The decoherence-free subalgebra of a quantum Markov semigroup with unbounded generator," Infin. Dimens. Anal. Quantum Probab. Relat. Top. 13, 413-433 (2010).

${ }^{17}$ K. J. Engel and R. Nagel, One-Parameter Semigroups for Linear Evolution Equations (Springer-Verlag, New York, 2000).

${ }^{18}$ D. E. Evans, "Irreducible quantum dynamical semigroups," Commun. Math. Phys. 54, 293-297 (1977).

${ }^{19}$ A. Frigerio, "Stationary states of quantum dynamical semigroups," Commun. Math. Phys. 63, 269-276 (1978).

${ }^{20}$ A. Frigerio and M. Verri, "Long-time asymptotic properties of dynamical semigroups on $W^{*}$-algebras," Math. Z. 180, 275-286 (1982).

${ }^{21}$ V. Gorini, A. Kossakowski, and E. C. G. Sudarshan, "Completely positive dynamical semigroups of N-level systems," J. Math. Phys. 17, 821-825 (1976).

${ }^{22}$ M. Hellmich, "Quantum dynamical semigroups and decoherence," Adv. Math. Phys. 2011, 625978.

${ }^{23}$ D. A. Lidar and K. B. Whaley, "Decoherence-free subspaces and subsystems," Lect. Notes Phys. 622, 83-120 (2003).

${ }^{24}$ G. Lindblad, "On the generators of quantum dynamical semigroups," Commun. Math. Phys. 48, 119-130 (1976).

${ }^{25}$ B. Kummerer, and R. Nagel, Mean ergodic semigroups on $W^{*}$-algebras. Acta Sci. Math. (Szeged) 41(1-2), 151-159 (1979).

${ }^{26}$ P. Lugiewicz and R. Olkiewicz, "Classical properties of infinite quantum open systems," Commun. Math. Phys. 239(1), 241-259 (2003).

${ }^{27}$ K. R. Parthasarathy, “An introduction to quantum stochastic calculus," in Monographs in Mathematics (Birkhäuser-Verlag, Basel, 1992), Vol. 85.

${ }^{28}$ M. Takesaki, "Conditional expectations in von Neumann algebras," J. Funct. Anal. 9, 306-321 (1972).

${ }^{29} \mathrm{M}$. Takesaki, Theory of Operator Algebras I (Springer-Verlag, New York; Heidelberg, 1979).

${ }^{30} \mathrm{~F}$. Ticozzi and L. Viola, “Quantum Markovian subsystems: Invariance, attractivity, and control," IEEE Trans. Autom. Control 53, 2048-2063 (2008).

${ }^{31}$ J. Tomiyama, "On the projection of norm one in $W^{*}$-algebras III," Tôhoku Math. J. 11, 125-129 (1959).

${ }^{32}$ M. M. Wolf, Quantum channel and operations, guided tour, notes, 2012. 